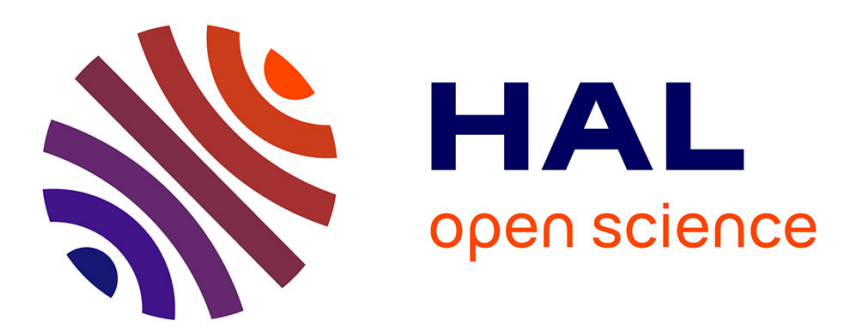

\title{
Willis elastodynamic homogenization theory revisited for periodic media
}

\author{
H. Nassar, Qi-Chang He, Nicolas Auffray
}

\section{To cite this version:}

H. Nassar, Qi-Chang He, Nicolas Auffray. Willis elastodynamic homogenization theory revisited for periodic media. Journal of the Mechanics and Physics of Solids, 2015, 77, pp.158-178. 10.1016/j.jmps.2014.12.011 . hal-01112307

\section{HAL Id: hal-01112307 \\ https://hal.science/hal-01112307}

Submitted on 2 Feb 2015

HAL is a multi-disciplinary open access archive for the deposit and dissemination of scientific research documents, whether they are published or not. The documents may come from teaching and research institutions in France or abroad, or from public or private research centers.
L'archive ouverte pluridisciplinaire HAL, est destinée au dépôt et à la diffusion de documents scientifiques de niveau recherche, publiés ou non, émanant des établissements d'enseignement et de recherche français ou étrangers, des laboratoires publics ou privés. 


\title{
Willis elastodynamic homogenization theory revisited for periodic media
}

\author{
Hussein Nassar, Qi-Chang He*, Nicolas Auffray \\ Université Paris-Est, Modélisation et Simulation Multi-Echelle, MSME UMR 8208 CNRS, \\ 5 bd Descartes, 77454 Marne-la-Vallée, France
}

\begin{abstract}
The theory of elastodynamic homogenization initiated by J.R. Willis is revisited for periodically inhomogeneous media through a careful scrutiny of the main aspects of that theory in the 3D continuum context and by applying it to the thorough treatment of a simple 1D discrete periodic system. The Bloch theorem appears to be central to appropriately defining and interpreting effective fields. Based on some physical arguments, three necessary conditions are derived for the transition from the microscopic description to the macroscopic description of periodic media. The parameters involved in the Willis effective constitutive relation are expressed in terms of two localization tensors and specified with the help of the corresponding Green function in the spirit of micromechanics. These results are illustrated and discussed for the 1D discrete periodic system considered. In particular, inspired by Brillouin's study, the dependency of the effective constitutive parameters on the frequency is physically interpreted in terms of oscillation modes of the underlying microstructure.
\end{abstract}

Keywords: Homogenization, Bloch waves, Dynamics, Constitutive behavior, Inhomogeneous material

\section{Introduction}

The beginnings of the elastodynamic homogenization theory of J.R. Willis can be traced back to the relevant papers he published during the first half of the 1980s (Willis, 1980a,b, 1981, 1985). The main body of this theory was, in a rather complete manner, presented more than 10 years later in a chapter of a book edited

\footnotetext{
${ }^{*}$ Corresponding author

Email addresses: hussein.nassar@univ-paris-est.fr (Hussein Nassar), qi-chang.he@univ-paris-est.fr (Qi-Chang He), nicolas.auffray@univ-paris-est.fr (Nicolas Auffray)
} 
after a course dedicated to continuum micromechanics (Willis, 1997). Recently, increasing interest in acoustic metamaterials and cloaking (see, e.g., papers by Chen and Chan (2010), Lee et al. (2012), Liu et al. (2000), Liu et al. (2012), Milton et al. (2006), Norris (2008), Norris and Shuvalov (2011) and Simovski (2007)) has, in particular, given an impetus to the development and application of the elastodynamic homogenization theory of Willis (Milton and Willis, 2007, 2010; Nemat-Nasser and Srivastava, 2011, 2013; Nemat-Nasser et al., 2011; Norris et al., 2012; Shuvalov et al., 2011; Srivastava and Nemat-Nasser, 2011; Willis, 2009, 2011, 2012).

The elastodynamic homogenization theory of Willis exhibits the following salient features: (i) in the microscopic-to-macroscopic upscaling process, no approximation hypotheses are made, so that, in this sense, the resulting theory can be considered as exact; (ii) the effects of material microscopic inhomogeneities are, after homogenization, all incorporated only in the resulting non-classical effective constitutive law, so that the macroscopic (or effective) motion equation takes the same classical form as the one at the microscopic level; (iii) for a composite formed of elastic phases whose constitutive laws are local in time and space, the effective constitutive law obtained by homogenization becomes generally nonlocal both in time and space; (iv) the effective mass density is, in general, no longer a scalar but a second-order tensor quantity; (v) a non-classical coupling between the effective stress tensor and the effective velocity, and another one between the effective momentum and the effective strain tensor, occur generally in the effective constitutive law; (vi) the parameters involved in the effective constitutive law are non-unique but can be rendered unique by prescribing, for example, an additional eigenstrain field. Note that the features (iii)-(vi) make that the effective constitutive law derived in the homogenized elastodynamic theory of Willis is very different from the constitutive law involved in the classical elastodynamic theory and that its explicit determination in terms of the phase properties is a quite tough task and in general necessitates using a numerical method.

The present work consists in revisiting the elastodynamic homogenization theory of Willis for periodic composites so as to reach the following threefold objective. First, it aims to derive, on the basis of some physically sound arguments, a few necessary conditions for the application of that theory to be physically meaningful. Second, it has the purpose of expressing the effective constitutive parameters of the effective constitutive law in terms of some appropriate localization tensors in the spirit of micromechanics, so that a general numerical method, such as the finite element method, can be directly used to numerically compute said parameters. Thirdly, it aims to gain physical insights into the general theory by applying it to thoroughly and analytically study a simple one-dimensional (1D) periodic discrete system. By achieving these three objectives, the present work contributes not 
only to getting a better understanding of but also developing the elastodynamic homogenization theory initiated by Willis.

The next sections of this paper are structured and summarized as follows.

In section 2, which is the main part of this paper, the elastodynamic homogenization theory of Willis is carefully reformulated mathematically and examined physically for periodic composites. After providing some geometrical preliminaries and recalling the local classical elastodynamic equations for periodic media, Bloch theorem is shown to play a central role in solving the corresponding local motion equation and in properly defining and interpreting the effective (or macroscopic) fields. Three necessary conditions are then proposed for the elastodynamic homogenization theory of Willis applied to a periodic composite to lead to a physically meaningful effective behavior. The first necessary condition corresponds to the requirement that the microscopic virtual work be equal to the effective (or macroscopic) virtual work, which is reminiscent of the well-known Hill-Mandel lemma in micromechanics. The second necessary condition concerns wavenumbers and demands that the effective (or macroscopic) fields capture the long-wavelength parts of the relevant microscopic fields. The third necessary condition is relative to frequencies and comes from the requirement that the effective elastodynamic behavior of a composite be a good approximation of its microscopic one. In the last part of section 2, two localization tensors are first introduced in the spirit of micromechanics and then explicitly expressed in terms of the relevant Green function for a given pair of frequency and wavenumber. With the help of the expressions for the localization tensors, the frequency- and wavenumber-dependent parameters characterizing the effective elastodynamic constitutive law are finally specified in terms of the Green function. The obtained effective elastodynamic behavior is then reinterpreted physically in light of the established homogenizability conditions.

In section 3, inspired by the work of Brillouin (1953), a simple 1D periodic discrete system is analytically and exhaustively studied to illustrate and discuss the main results of section 2. In particular, the effective constitutive parameters are analytically and exactly obtained, and the effective impedance and dispersion relation are derived and illustrated. The homogenizability conditions proposed in section 2 are discussed.

Finally, in section 4, a few concluding remarks are drawn and some open problems are mentioned.

\section{Elements of an elastodynamic homogenization theory for periodic media}

We start by presenting some useful geometrical tools to the study of periodic media and the basic motion and constitutive equations. The motion equation is 
subsequently simplified and restricted to one arbitrary unit cell thanks to BlochFloquet theorem. The Bloch-wave-expansion leads to a definition of the effective fields and the corresponding effective behavior. We then thoroughly discuss the consistency of the Willis approach according to three criteria hereafter called "homogenizability conditions". Such a discussion is possible even before having any expression of the effective constitutive equations. The derivation of said expression is presented last.

\subsection{Problem set-up}

\subsubsection{Geometrical considerations}

Let $\Omega$ be a periodic medium. We liken $\Omega$ to a 3D point space with an underlying vector space called $\mathscr{E}$ and a periodicity lattice $\mathscr{R}$. Vectors of $\mathscr{E}$ can be identified with points of $\Omega$ through the choice of an arbitrary origin. The lattice $\mathscr{R}$ is defined as

$$
\mathscr{R}=\left\{\boldsymbol{r} \in \mathscr{E} \mid \boldsymbol{r}=a_{1} \boldsymbol{b}_{1}+a_{2} \boldsymbol{b}_{2}+a_{3} \boldsymbol{b}_{3}, a_{i} \in \mathbb{Z}\right\}
$$

where $\mathbb{Z}$ stands for the set of integers and the vectors $\left(\boldsymbol{b}_{1}, \boldsymbol{b}_{2}, \boldsymbol{b}_{3}\right)$ form a basis for $\mathscr{E}$. A unit cell $T$ for $\Omega$ can then be specified as

$$
T=\left\{\boldsymbol{r} \in \mathscr{E} \mid \boldsymbol{r}=r_{1} \boldsymbol{b}_{1}+r_{2} \boldsymbol{b}_{2}+r_{3} \boldsymbol{b}_{3}, r_{i} \in[0,1[\} .\right.
$$

Note that neither the choice of $\left(\boldsymbol{b}_{1}, \boldsymbol{b}_{2}, \boldsymbol{b}_{3}\right)$ nor that of $T$ is unique. In figure 1 , a two-dimensional (2D) lattice $\mathscr{R}$ is illustrated.

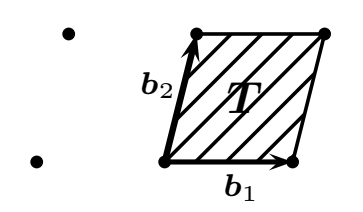

Figure 1: A 2D lattice. Each point represents a vector $\boldsymbol{r}$ of the lattice $\mathscr{R}$. Many choices for $\boldsymbol{b}_{1}$, $\boldsymbol{b}_{2}$ and $T$ are possible.

The vector space dual to $\mathscr{E}$ is symbolized by $\mathscr{E}^{*}$. In what follows, $\mathscr{E} *$ corresponds physically to the space of wavenumbers, and a generic element of $\mathscr{E}^{*}$ is denoted by $\boldsymbol{k}$. For later use, it is useful to introduce the reciprocal lattice $\mathscr{R}^{*}$ of $\mathscr{R}$ :

$$
\mathscr{R}^{*}=\left\{\boldsymbol{\xi} \in \mathscr{E}^{*} \mid \boldsymbol{\xi}=a_{1} \boldsymbol{b}_{1}^{*}+a_{2} \boldsymbol{b}_{2}^{*}+a_{3} \boldsymbol{b}_{3}^{*}, a_{i} \in \mathbb{Z}\right\} .
$$

\footnotetext{
${ }^{1}$ The superscript $\left(^{*}\right)$ will mean reciprocal, dual or conjugate depending on the objects it acts on; each new meaning of this notation will be defined precisely when first used.
} 
In this definition, the vectors $\left(\boldsymbol{b}_{1}^{*}, \boldsymbol{b}_{2}^{*}, \boldsymbol{b}_{3}^{*}\right)$ form a basis for $\mathscr{E}^{*}$ and verify

$$
\boldsymbol{b}_{i}^{*} \cdot \boldsymbol{b}_{j}=2 \pi \delta_{i j}
$$

where the dot operator $(\cdot)$ stands for the scalar product, $\delta_{i j}$ is the Kronecker delta and $2 \pi$ is a conventional scaling factor. In addition, use will be made of the first Brillouin zone $T^{*}$ of the reciprocal lattice $\mathscr{R}^{*}$ :

$$
T^{*}=\left\{\boldsymbol{k} \in \mathscr{E}^{*} \mid \forall \boldsymbol{\xi} \in \mathscr{R}^{*}-\{\mathbf{0}\},\|\boldsymbol{k}\|<\|\boldsymbol{k}-\boldsymbol{\xi}\|\right\} .
$$

We can geometrically interpret $T^{*}$ as the set of wavenumbers $\boldsymbol{k}$ of $\mathscr{E}^{*}$ which are closer to the null wavenumber than to any other wavenumber $\boldsymbol{\xi}$ of the reciprocal lattice $\mathscr{R}^{*}$. It should be underlined that the interior of $T^{*}$ is uniquely defined and is, in particular, independent of the choice of $T$. In figure 2 , a $2 \mathrm{D}$ example for $\mathscr{R}^{*}$ and $T^{*}$ is shown.

Given a function $\phi(\boldsymbol{x})$ of the space variable $\boldsymbol{x} \in \mathscr{E}$, its spatial Fourier transform $\phi_{\boldsymbol{k}}$ is defined over the space $\mathscr{E}^{*}$ of wavenumbers $\boldsymbol{k}$ by

$$
\phi(\boldsymbol{x})=\int_{\mathscr{E}^{*}} \phi_{k} e^{i \boldsymbol{k} \cdot \boldsymbol{x}} \mathrm{d} \boldsymbol{k},
$$

where $i^{2}=-1$. In particular, an $\mathscr{R}$-periodic function $\phi(\boldsymbol{x})$ can be expanded into the Fourier series

$$
\phi(\boldsymbol{x})=\sum_{\boldsymbol{\xi} \in \mathscr{R}^{*}} \phi_{\xi} e^{i \xi \cdot \boldsymbol{x}}
$$

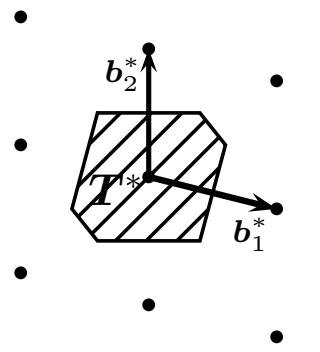

Figure 2: The reciprocal lattice $\mathscr{R}^{*}$ of the direct lattice $\mathscr{R}$ depicted in figure 1 and its first Brillouin zone $T^{*}$.

\footnotetext{
${ }^{3}$ Vectors $\boldsymbol{b}_{i}$ and $\boldsymbol{b}_{i}^{*}$ have different dimensions (respectively, a length and the inverse of a length) and their magnitudes, therefore, cannot be compared.
} 


\subsubsection{Local elastodynamic equations}

The periodic medium $\Omega$ under consideration is assumed to undergo small deformations and behave as a linearly elastic inhomogeneous material. Aiming to carry out the elastodynamic homogenization of $\Omega$, we first recall the relevant local equations.

Letting $\boldsymbol{u}$ be the displacement field over $\Omega$, the infinitesimal strain field $\varepsilon$ is given by

$$
\boldsymbol{\varepsilon}=\frac{1}{2}(\boldsymbol{\nabla} \otimes \boldsymbol{u}+\boldsymbol{u} \otimes \nabla) \equiv \boldsymbol{\nabla} \otimes^{s} \boldsymbol{u}
$$

where $\boldsymbol{\nabla}$ is the gradient operator, the symbol $\otimes$ designates the tensor product, the superscript $\left({ }^{s}\right)$ stands for symmetrization and the relation $\equiv$ is equality by definition. The stress field $\boldsymbol{\sigma}$ and the (linear) momentum field $\boldsymbol{p}$ are related to the strain field $\varepsilon$ and the velocity field $\boldsymbol{v}$ by

$$
\boldsymbol{\sigma}=\boldsymbol{C}: \boldsymbol{\varepsilon}, \quad \boldsymbol{p}=\rho \boldsymbol{v}
$$

where the colon (:) stands for double contraction, $\boldsymbol{C}$ is the fourth-order elastic stiffness tensor and $\rho$ is the mass density. Owing to the periodicity of $\Omega$, we have

$$
\boldsymbol{C}(\boldsymbol{x}+\boldsymbol{r})=\boldsymbol{C}(\boldsymbol{x}), \quad \rho(\boldsymbol{x}+\boldsymbol{r})=\rho(\boldsymbol{x}),
$$

for every point $\boldsymbol{x} \in \Omega$ and all lattice vectors $\boldsymbol{r} \in \mathscr{R}$. Thus, the elastic and inertial properties of $\Omega$ are entirely defined by specifying them over a unit cell $T$ and by periodicity. Let $f$ be a body force field over $\Omega$, then the motion equation of $\Omega$ takes the form

$$
\nabla \cdot \sigma+f=\dot{p}
$$

with $(\boldsymbol{\nabla} \cdot)$ being the divergence operator and the superimposed dot standing for the time derivative ${ }^{4}$.

The problem to be treated in what follows is the homogenization of the periodically heterogeneous medium forming $\Omega$ within the framework of elastodynamics. Precisely, that periodically heterogeneous medium will be replaced by a homogeneous medium over which appropriate effective fields are defined and whose

\footnotetext{
${ }^{4}$ One can account for initial displacement and velocity conditions by changing the unknown displacement field:

$$
\boldsymbol{u}(\boldsymbol{x}, t) \mapsto H(t) \boldsymbol{u}(\boldsymbol{x}, t)
$$

where $H(t)$ is the Heaviside function, and correspondingly altering the body force:

$$
\boldsymbol{f}(\boldsymbol{x}, t) \mapsto \boldsymbol{f}(\boldsymbol{x}, t)+\delta(t) \rho(\boldsymbol{x}) \dot{\boldsymbol{u}}(\boldsymbol{x}, t=0)+\dot{\delta}(t) \rho(\boldsymbol{x}) \boldsymbol{u}(\boldsymbol{x}, t=0)
$$

where $\delta(t)$ is the Dirac function. Initial conditions can then be thought of as a particular loading applied at $t=0$. However, we shall mostly work with harmonic fields.
} 
elastodynamic behavior is determined in such a way that the most important elastodynamic features of the initial periodic medium are captured. To achieve this objective, we shall work with transformed fields instead of dealing directly with fields of space and time. As will be seen, the Bloch theorem and the superposition principle pave a very efficient method.

\subsection{Bloch-wave-expansion and simplified motion equation}

We start by performing the partial Fourier transform of the local motion equation with respect to time:

$$
\boldsymbol{\nabla} \cdot \boldsymbol{\sigma}_{\omega}(\boldsymbol{x})+\boldsymbol{f}_{\omega}(\boldsymbol{x})=i \omega \boldsymbol{p}_{\omega}(\boldsymbol{x})
$$

where $\omega$ is the time angular frequency, simply referred to as the frequency, and the notation $\phi_{\omega}(\boldsymbol{x})$ stands for the time-Fourier transform of $\boldsymbol{\phi}(\boldsymbol{x}, t)$ satisfying

$$
\phi(\boldsymbol{x}, t)=\int_{\mathbb{R}} \phi_{\omega}(\boldsymbol{x}) e^{i \omega t} \mathrm{~d} \omega .
$$

One way to determine the displacement response $\boldsymbol{u}_{\omega}(\boldsymbol{x})$ to the solicitation $\boldsymbol{f}_{\omega}(\boldsymbol{x})$ is to calculate elementary responses to elementary plane wave solicitations. Letting $\boldsymbol{f}_{\boldsymbol{k}, \omega}$ be the spatial Fourier transform of the harmonic force field $\boldsymbol{f}_{\omega}(\boldsymbol{x})$, we have the plane-wave-expansion of $\boldsymbol{f}_{\omega}(\boldsymbol{x})$ as follows:

$$
\boldsymbol{f}_{\omega}(\boldsymbol{x})=\int_{\mathscr{E}^{*}} \boldsymbol{f}_{\boldsymbol{k}, \omega} e^{i \boldsymbol{k} \cdot \boldsymbol{x}} \mathrm{d} \boldsymbol{k},
$$

so that the phase of body force is $\boldsymbol{k} \cdot \boldsymbol{x}+\omega t$ with a plus sign convention. We denote by $\boldsymbol{u}_{\boldsymbol{k}, \omega}(\boldsymbol{x}) e^{i \boldsymbol{k} \cdot \boldsymbol{x}}$ the elementary displacement response of $\Omega$ to the plane wave solicitation $\boldsymbol{f}_{\boldsymbol{k}, \omega} e^{i \boldsymbol{k} \cdot \boldsymbol{x}}$. This response is a solution of the equation

$$
\boldsymbol{\nabla} \cdot\left\{\boldsymbol{C}(\boldsymbol{x}):\left[\boldsymbol{\nabla} \otimes^{s}\left(\boldsymbol{u}_{\boldsymbol{k}, \omega}(\boldsymbol{x}) e^{i \boldsymbol{k} \cdot \boldsymbol{x}}\right)\right]\right\}+\boldsymbol{f}_{\boldsymbol{k}, \omega} e^{i \boldsymbol{k} \cdot \boldsymbol{x}}=-\omega^{2} \rho(\boldsymbol{x}) \boldsymbol{u}_{\boldsymbol{k}, \omega}(\boldsymbol{x}) e^{i \boldsymbol{k} \cdot \boldsymbol{x}}
$$

At a given frequency $\omega$, free wave solutions ${ }^{5}$ have specific wavelengths. In what follows, we exclude such wavelengths so as to guarantee the solution uniqueness. Next, consider the foregoing problem translated by a lattice vector $\boldsymbol{r} \in \mathscr{R}$ :

$$
\begin{aligned}
\boldsymbol{\nabla} \cdot\left\{\boldsymbol{C}(\boldsymbol{x}+\boldsymbol{r}):\left[\boldsymbol{\nabla} \otimes^{s}\left(\boldsymbol{u}_{\boldsymbol{k}, \omega}(\boldsymbol{x}+\boldsymbol{r}) e^{i \boldsymbol{k} \cdot(\boldsymbol{x}+\boldsymbol{r})}\right)\right]\right\}+\boldsymbol{f}_{\boldsymbol{k}, \omega} e^{i \boldsymbol{k} \cdot(\boldsymbol{x}+\boldsymbol{r})} \\
=-\omega^{2} \rho(\boldsymbol{x}+\boldsymbol{r}) \boldsymbol{u}_{\boldsymbol{k}, \omega}(\boldsymbol{x}+\boldsymbol{r}) e^{i \boldsymbol{k} \cdot(\boldsymbol{x}+\boldsymbol{r})}
\end{aligned}
$$

We cancel the factor $e^{i \boldsymbol{k} \cdot \boldsymbol{r}}$ and apply the $\mathscr{R}$-periodicity to $\boldsymbol{C}$ and to $\rho$ in the above equation and obtain

$$
\boldsymbol{\nabla} \cdot\left\{\boldsymbol{C}(\boldsymbol{x}):\left[\boldsymbol{\nabla} \otimes^{s}\left(\boldsymbol{u}_{\boldsymbol{k}, \omega}(\boldsymbol{x}+\boldsymbol{r}) e^{i \boldsymbol{k} \cdot \boldsymbol{x}}\right)\right]\right\}+\boldsymbol{f}_{\boldsymbol{k}, \omega} e^{i \boldsymbol{k} \cdot \boldsymbol{x}}=-\omega^{2} \rho(\boldsymbol{x}) \boldsymbol{u}_{\boldsymbol{k}, \omega}(\boldsymbol{x}+\boldsymbol{r}) e^{i \boldsymbol{k} \cdot \boldsymbol{x}}
$$

\footnotetext{
${ }^{5}$ Solutions of $\boldsymbol{\nabla} \cdot \boldsymbol{\sigma}_{\omega}(\boldsymbol{x})=i \omega \boldsymbol{p}_{\omega}(\boldsymbol{x})$.
} 
Therefore, $\boldsymbol{u}_{\boldsymbol{k}, \omega}(\boldsymbol{x}) e^{i \boldsymbol{k} \cdot \boldsymbol{x}}$ and $\boldsymbol{u}_{\boldsymbol{k}, \omega}(\boldsymbol{x}+\boldsymbol{r}) e^{i \boldsymbol{k} \cdot \boldsymbol{x}}$ turn out to be two solutions for the same problem. The solution uniqueness allows us to conclude that $\boldsymbol{u}_{k, \omega}(\boldsymbol{x})=\boldsymbol{u}_{\boldsymbol{k}, \omega}(\boldsymbol{x}+\boldsymbol{r})$ for all lattice vectors $\boldsymbol{r} \in \mathscr{R}$. The elementary solution then admits the so-called Bloch form

$$
\boldsymbol{u}_{\boldsymbol{k}, \omega}(\boldsymbol{x}) e^{i \boldsymbol{k} \cdot \boldsymbol{x}} \quad \text { where } \quad \boldsymbol{u}_{\boldsymbol{k}, \omega}(\boldsymbol{x}) \text { is } \mathscr{R} \text {-periodic. }
$$

For the excluded free wave solutions, the same Bloch form still hold. A proof in this case can be found in a paper by Gazalet et al. (2013) (see also Appendix A).

To sum up, the solution $\boldsymbol{u}(\boldsymbol{x}, t)$ of the motion equation can be constructed as a superposition of harmonic-Bloch waves, indexed by $(\boldsymbol{k}, \omega)$, of the form $\boldsymbol{u}_{\boldsymbol{k}, \omega}(\boldsymbol{x}) e^{i(\boldsymbol{k} \cdot \boldsymbol{x}+\omega t)}$ with $\boldsymbol{u}_{k, \omega}(\boldsymbol{x})$ being $\mathscr{R}$-periodic. The couple $(\boldsymbol{k}, \omega)$ is given once and for all; the dependencies relative to time and to $(\boldsymbol{k}, \omega)$ will often be omitted. The elementary body force field will be denoted simply by $\boldsymbol{f}(\boldsymbol{x})=\tilde{\boldsymbol{f}} e^{i \boldsymbol{k} \cdot \boldsymbol{x}}$ where $\tilde{\boldsymbol{f}}$ is independent of $\boldsymbol{x}$. All other fields $\boldsymbol{\phi} \in\{\boldsymbol{u}, \boldsymbol{v}, \boldsymbol{\varepsilon}, \boldsymbol{p}, \boldsymbol{\sigma}\}$ have the Bloch form $\boldsymbol{\phi}(\boldsymbol{x})=\tilde{\boldsymbol{\phi}}(\boldsymbol{x}) e^{i \boldsymbol{k} \cdot \boldsymbol{x}}$ with $\tilde{\phi}(\boldsymbol{x})$ being $\mathscr{R}$-periodic. The motion equation, in terms of $\tilde{\boldsymbol{u}}$,

$$
\boldsymbol{\nabla} \cdot\left\{\boldsymbol{C}(\boldsymbol{x}):\left[\boldsymbol{\nabla} \otimes^{s}\left(\tilde{\boldsymbol{u}}(\boldsymbol{x}) e^{i \boldsymbol{k} \cdot \boldsymbol{x}}\right)\right]\right\}+\tilde{\boldsymbol{f}} e^{i \boldsymbol{k} \cdot \boldsymbol{x}}=-\omega^{2} \rho(\boldsymbol{x}) \tilde{\boldsymbol{u}}(\boldsymbol{x}) e^{i \boldsymbol{k} \cdot \boldsymbol{x}}
$$

or equivalently, by expanding the derivatives of $e^{i \boldsymbol{k} \cdot \boldsymbol{x}}$,

$$
(\boldsymbol{\nabla}+i \boldsymbol{k}) \cdot\left\{\boldsymbol{C}:\left[(\boldsymbol{\nabla}+i \boldsymbol{k}) \otimes^{s} \tilde{\boldsymbol{u}}\right]\right\}+\tilde{\boldsymbol{f}}=-\omega^{2} \rho \tilde{\boldsymbol{u}}
$$

needs then to be solved only over the unit cell $T$, under periodic boundary conditions.

\subsection{Effective fields and effective motion equation}

Following Amirkhizi and Nemat-Nasser (2008), we define the effective field $\boldsymbol{\Phi}$ of a microscopic Bloch-wave field $\phi(\boldsymbol{x})=\tilde{\boldsymbol{\phi}}(\boldsymbol{x}) e^{i \boldsymbol{k} \cdot \boldsymbol{x}}$ as being the plane wave

$$
\boldsymbol{\Phi}(\boldsymbol{x})=\frac{1}{|T|}\left(\int_{T} \tilde{\boldsymbol{\phi}}(\boldsymbol{y}) \mathrm{d} \boldsymbol{y}\right) e^{i \boldsymbol{k} \cdot \boldsymbol{x}} \equiv\langle\tilde{\boldsymbol{\phi}}\rangle e^{i \boldsymbol{k} \cdot \boldsymbol{x}}
$$

where $|T|$ is the volume of $T$ and $\langle\cdot\rangle$ is the space-average-over- $T$ operator. We insist on the fact that only $\mathscr{R}$-periodic fields (e.g., $\tilde{\phi})$ can be averaged without any ambiguity as all choices of $T$ are equivalent. A non $\mathscr{R}$-periodic field will have different averages over different unit cells. Definition (2.3) is equivalent to Willis (2011) ensemble average: at a point $\boldsymbol{x}, \boldsymbol{\Phi}(\boldsymbol{x})$ is the expected value of multiple measures $\phi(\boldsymbol{x})$ recovered from multiple experiences carried out over multiple samples taken randomly out of the same material.

For the moment, we argue that the effective field definition (2.3) allows to satisfy exactly the usual motion equation. We recast (2.2) into the equivalent form

$$
\nabla \cdot \tilde{\boldsymbol{\sigma}}+i \boldsymbol{k} \cdot \tilde{\boldsymbol{\sigma}}+\tilde{\boldsymbol{f}}=i \omega \tilde{\boldsymbol{p}} .
$$


Then, by the divergence theorem and the periodic boundary conditions, averaging over $T$ yields

$$
i \boldsymbol{k} \cdot\langle\tilde{\boldsymbol{\sigma}}\rangle+\langle\tilde{\boldsymbol{f}}\rangle=i \omega\langle\tilde{\boldsymbol{p}}\rangle .
$$

When multiplied by $e^{i \boldsymbol{k} \cdot \boldsymbol{x}}$, the above equation becomes the effective motion equation

$$
\boldsymbol{\nabla} \cdot \boldsymbol{\Sigma}+\boldsymbol{F}=i \omega \boldsymbol{P}
$$

with $\boldsymbol{F}=\boldsymbol{f}$. Therefore, the effective medium satisfies the same motion equation as for the microscopic medium, and the body force remains unchanged.

\subsection{Necessary conditions for homogenization}

For the effective fields defined in (2.3) to act as physically meaningful macroscopic fields, frequencies and wavenumbers have to satisfy some conditions, hereafter called homogenizability conditions. Below, we derive three of them. The first one amounts to a generalized version of Hill-Mandel lemma. The second one is based on the physically sound requirement that the effective field $\boldsymbol{\Phi}$ defined for a microscopic field $\phi$ be a slowly space-varying approximation of $\phi$. The third one comes from the demand that $\boldsymbol{\Phi}$ be a good approximation of $\phi$.

\subsubsection{First condition: Hill-Mandel lemma}

Starting from the microscopic and effective motion equations (2.2) and (2.4), we can show that the virtual work theorem holds, so that ${ }^{6}$

$$
\begin{aligned}
\left\langle\boldsymbol{f} \cdot \boldsymbol{u}^{*}\right\rangle & =\left\langle\boldsymbol{\sigma}: \varepsilon^{*}+i \omega \boldsymbol{p} \cdot \boldsymbol{u}^{*}\right\rangle, \\
\left\langle\boldsymbol{F} \cdot \boldsymbol{U}^{*}\right\rangle & =\left\langle\boldsymbol{\Sigma}: \boldsymbol{E}^{*}+i \omega \boldsymbol{P} \cdot \boldsymbol{U}^{*}\right\rangle
\end{aligned}
$$

where the superscript star $\left(^{*}\right)$ stands for complex conjugation. The following straightforward calculation

$$
\left\langle\boldsymbol{f} \cdot \boldsymbol{u}^{*}\right\rangle=\left\langle\tilde{\boldsymbol{f}} \cdot \tilde{\boldsymbol{u}}^{*}\right\rangle=\tilde{\boldsymbol{f}} \cdot\left\langle\tilde{\boldsymbol{u}}^{*}\right\rangle=\tilde{\boldsymbol{f}} e^{i \boldsymbol{k} \cdot \boldsymbol{x}} \cdot\left(\langle\tilde{\boldsymbol{u}}\rangle e^{i \boldsymbol{k} \cdot \boldsymbol{x}}\right)^{*}=\boldsymbol{F} \cdot \boldsymbol{U}^{*}=\left\langle\boldsymbol{F} \cdot \boldsymbol{U}^{*}\right\rangle
$$

shows that both microscopic and effective works are equal to each other. Therefore, we have

$$
\left\langle\boldsymbol{\sigma}: \varepsilon^{*}+i \omega \boldsymbol{p} \cdot \boldsymbol{u}^{*}\right\rangle=\left\langle\boldsymbol{\Sigma}: \boldsymbol{E}^{*}+i \omega \boldsymbol{P} \cdot \boldsymbol{U}^{*}\right\rangle,
$$

which is reminiscent of the well-known Hill-Mandel lemma. Even though products such as $\boldsymbol{\Sigma}: \boldsymbol{E}^{*}$ are constant in space, it is important to recall that all effective fields are $\boldsymbol{x}$-dependent. Finally, neither the microscopic constitutive equation, nor the

\footnotetext{
${ }^{6}$ using integration by parts and divergence theorem while accounting for the fact that boundary terms are null owing to the Bloch form.
} 
(to-be-found) effective one has been used to establish the above equality, therefore, Hill-Mandel lemma is valid for virtual fields independently of any constitutive law.

It is of interest to see whether the lemma (2.5) holds or not for combinations of Bloch waves. It is enough to consider two sets of microscopic and effective fields $\boldsymbol{\phi}, \boldsymbol{\Phi}$ and $\boldsymbol{\phi}^{\prime}, \boldsymbol{\Phi}^{\prime}$ corresponding to two modes $(\omega, \boldsymbol{k})$ and $\left(\omega, \boldsymbol{k}^{\prime}\right)$. The question is:

$$
\left\langle\left(\boldsymbol{f}+\boldsymbol{f}^{\prime}\right) \cdot\left(\boldsymbol{u}+\boldsymbol{u}^{\prime}\right)^{*}\right\rangle \stackrel{?}{=}\left\langle\left(\boldsymbol{F}+\boldsymbol{F}^{\prime}\right) \cdot\left(\boldsymbol{U}+\boldsymbol{U}^{\prime}\right)^{*}\right\rangle .
$$

The averaged quantities here are unfortunately non-periodic. Therefore, before answering the above question, we need to extend the definition of the averaging operator to non-periodic fields. This is done according to:

$$
\langle\cdot\rangle=\lim _{R \rightarrow+\infty} \frac{1}{|B(R)|} \int_{B(R)} \cdot \mathrm{d} \boldsymbol{x}
$$

where $B(R)$ is a ball of radius $R$ and $|B(R)|$ is its volume. For $\mathscr{R}$-periodic fields, the above definition yields exactly the usual average over one unit cell $T$. Remark also that for any wavenumber $\boldsymbol{k} \in \mathscr{E}^{*}-\{\mathbf{0}\},\left\langle e^{i \boldsymbol{k} \cdot \boldsymbol{x}}\right\rangle=0$.

Back to the question (2.6), it is elementary to prove that:

$$
\begin{aligned}
\left\langle\left(\boldsymbol{f}+\boldsymbol{f}^{\prime}\right) \cdot\left(\boldsymbol{u}+\boldsymbol{u}^{\prime}\right)^{*}\right\rangle-\left\langle\left(\boldsymbol{F}+\boldsymbol{F}^{\prime}\right) \cdot\left(\boldsymbol{U}+\boldsymbol{U}^{\prime}\right)^{*}\right\rangle & \\
& =\tilde{\boldsymbol{f}} \cdot\left\langle\tilde{\boldsymbol{u}}^{\prime} e^{i\left(\boldsymbol{k}^{\prime}-\boldsymbol{k}\right) \cdot \boldsymbol{x}}\right\rangle^{*}+\tilde{\boldsymbol{f}}^{\prime} \cdot\left\langle\tilde{\boldsymbol{u}} e^{i\left(\boldsymbol{k}-\boldsymbol{k}^{\prime}\right) \cdot \boldsymbol{x}}\right\rangle^{*},
\end{aligned}
$$

and that the error term in the right member always vanishes if and only if $\boldsymbol{k}-\boldsymbol{k}^{\prime} \notin \mathscr{R}^{*}$. Otherwise, $\boldsymbol{f}$ of wavenumber $\boldsymbol{k}$ (respectively, $\boldsymbol{f}^{\prime}$ of wavenumber $\boldsymbol{k}^{\prime}$ ) will work with some component of $\tilde{\boldsymbol{u}}^{\prime}$ of wavenumber $\boldsymbol{k}-\boldsymbol{k}^{\prime} \in \mathscr{R}^{*}$ (respectively, of $\tilde{\boldsymbol{u}}$ of wavenumber $\boldsymbol{k}^{\prime}-\boldsymbol{k} \in \mathscr{R}^{*}$ ) leading to violation of Hill-Mandel lemma.

To sum up, Hill-Mandel lemma (2.5) remains valid for combinations of planewave, and therefore arbitrary, loadings $\boldsymbol{f}$ as long as the Fourier spectrum of $\boldsymbol{f}, S_{\boldsymbol{f}}$, satisfies the following first homogenizability condition:

$$
\forall \boldsymbol{\xi} \in \mathscr{R}^{*}, \text { if } \boldsymbol{k} \in S_{\boldsymbol{f}} \text { then }(\boldsymbol{k}+\boldsymbol{\xi}) \notin S_{\boldsymbol{f}} .
$$

One example of such maximal spectra is the first Brillouin zone $T^{*}$. Note that this first condition has no implications on free waves propagation (for which $\boldsymbol{f}=\mathbf{0}$ ).

Hill-Mandel lemma as presented in equation (2.5) and generalized to arbitrary loadings satisfying condition (2.8) deals with the effective and microscopic virtual works and not with their expectancies. Willis (1997) proved another version of Hill-Mandel lemma based on the expectancies (the ensemble average) of virtual works, which is valid for all sure body forces. In fact, randomizing equation (2.7) then taking its expectancy yields a zero error term. Incidentally, this ensemble averaged version of Hill-Mandel lemma has been obtained somehow differently, but in an equivalent manner to Willis, by Smyshlyaev and Cherednichenko (2000). 


\subsubsection{Second condition: effective vs macroscopic}

As $\tilde{\phi}$ is $\mathscr{R}$-periodic, it admits a Fourier series expansion with respect to the reciprocal lattice $\mathscr{R}^{*}$ of the direct lattice $\mathscr{R}$. Thus,

$$
\boldsymbol{\phi}(\boldsymbol{x})=\tilde{\boldsymbol{\phi}}(\boldsymbol{x}) e^{i \boldsymbol{k} \cdot \boldsymbol{x}}=\sum_{\boldsymbol{\xi} \in \mathscr{R}^{*}} \tilde{\boldsymbol{\phi}}_{\xi} e^{i \boldsymbol{\xi} \cdot \boldsymbol{x}} e^{i \boldsymbol{k} \cdot \boldsymbol{x}}=\sum_{\boldsymbol{\xi} \in \mathscr{R}^{*}} \tilde{\boldsymbol{\phi}}_{\xi} e^{i(\boldsymbol{\xi}+\boldsymbol{k}) \cdot \boldsymbol{x}} .
$$

The slowest/long-wavelength component of $\phi$ is then given by

$$
\boldsymbol{\phi}^{\text {slow }}(\boldsymbol{x})=\tilde{\boldsymbol{\phi}}_{\zeta} e^{i(\boldsymbol{\zeta}+\boldsymbol{k}) \cdot \boldsymbol{x}}
$$

where $\boldsymbol{\zeta}$ corresponds to the vector of $\mathscr{R}^{*}$ minimizing the spatial frequency $\|\boldsymbol{\xi}+\boldsymbol{k}\|$ for $\boldsymbol{\xi} \in \mathscr{R}^{*}$. The effective description is a macroscopic one, in the sense that it corresponds to the behavior "as seen from far away", if it keeps track of the long-wavelength component. The second homogenizability condition consists in requiring

$$
\Phi=\phi^{\text {slow }} .
$$

This is equivalent to the proposition that

$$
\boldsymbol{\zeta}=\mathbf{0} \text { minimizes }\|\boldsymbol{\xi}+\boldsymbol{k}\| \text { for } \boldsymbol{\xi} \in \mathscr{R}^{*},
$$

because then $\tilde{\phi}_{\zeta}=\tilde{\phi}_{0}=\langle\tilde{\phi}\rangle$. Wavenumbers $\boldsymbol{k}$ which satisfy $\boldsymbol{\Phi}=\boldsymbol{\phi}^{\text {slow }}$ lie, by definition, in the first Brillouin zone:

$$
\boldsymbol{k} \in T^{*}
$$

If the body force $\boldsymbol{f}$ (including initial-conditions-related loadings) contains components with $\boldsymbol{k}$ outside of $T^{*}$, the effective fields as defined by equation (2.3) can no longer be interpreted as the long-wavelength part of the microscopic fields. Definition (2.3) can then be replaced by (2.9) with the purpose of saving the above second homogenizability condition. However, for the Hill-Mandel lemma (2.5) and the effective motion equation (2.4) to remain valid for this new definition, one must require (2.10) to hold. In other words: both definitions have to be identical so that the first one guarantees Hill-Mandel lemma and the effective motion equation and that the second one guarantees the validity of the long-wavelength-component interpretation. Remark at last that the present second homogenizability condition automatically implies the first one (2.8).

For example, back in the real space domain, body forces such as $\boldsymbol{f}(\boldsymbol{x})=$ $\rho(\boldsymbol{x}) \boldsymbol{f}^{\circ}$, where $\boldsymbol{f}^{\circ}$ is a homogeneous field, will violate condition (2.10). Such expression for body forces have been reported elsewhere to lead to a force-dependent effective behavior (Boutin, 1996). Here, the effective behavior will always be force-independent, however, short-wavelength body forces can lead to the above mentioned inconsistencies. 
For a 1D medium, if $|T|$ is the length of the unit cell $T$, the condition (2.10) reduces simply to

$$
-\frac{\pi}{|T|}<k<\frac{\pi}{|T|}
$$

Therefore, the effective field wavelength $\lambda=\left|\frac{2 \pi}{k}\right|$ must be at least twice as large as $T$ (see figure 3 ).

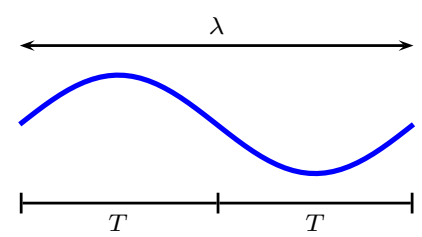

Figure 3: An illustration of the second homogenizability condition showing the shortest allowed wavelength.

\subsubsection{Third condition: on free waves}

The effective behavior is defined with respect to forced regime solutions. However, it is of interest to discuss some non negligible complications which arise for unforced regimes. Up till now, the wavenumber $\boldsymbol{k}$ has been prescribed by body forces which are null for free waves. Two remarks are therefore in order.

1. A microscopic solution $\boldsymbol{u}$ is transformed into an effective one $\boldsymbol{U}$ under the same loading and of the same mode $(\boldsymbol{k}, \omega)$. In particular, free waves are transformed into free waves and if a mode $(\boldsymbol{k}, \omega)$ allows for propagation of microscopic free waves, it also allows for propagation of effective ones.

2. A microscopic free wave $\boldsymbol{u}$ has multiple representations, one for each $\boldsymbol{\xi} \in \mathscr{R}^{*}$, as a Bloch wave:

$$
\boldsymbol{u}(\boldsymbol{x})=\tilde{\boldsymbol{u}}(\boldsymbol{x}) e^{i \boldsymbol{k} \cdot \boldsymbol{x}}=\left(\tilde{\boldsymbol{u}}(\boldsymbol{x}) e^{-i \boldsymbol{\xi} \cdot \boldsymbol{x}}\right) e^{i \boldsymbol{k}^{\prime} \cdot \boldsymbol{x}}
$$

with $\boldsymbol{k}^{\prime}=\boldsymbol{k}+\boldsymbol{\xi}$. In fact, the wavenumber of a free wave is only well defined modulo $\mathscr{R}^{*}\left(\bmod \mathscr{R}^{*}\right)$. The consequence is such that definition $(2.3)$ is ill posed for free waves. Given $\boldsymbol{u}(\boldsymbol{x})$ of mode $\left(\boldsymbol{k} \bmod \mathscr{R}^{*}, \omega\right)$, one can define multiple corresponding effective free waves of modes $(\boldsymbol{k}+\boldsymbol{\xi}, \omega)$ which are different from one another (each one corresponds to one Fourier component $\tilde{\boldsymbol{u}}_{\xi}$ of $\left.\tilde{\boldsymbol{u}}\right)$.

One needs then to precise which of the modes $(\boldsymbol{k}+\boldsymbol{\xi}, \omega)$ is (are) kept and which is (are) rejected. In other words, one needs to extend definition (2.3) unambiguously to free waves. Next, we discuss three possibilities. 
1. We can define the effective free wave as the limit of a forced one by a plane wave loading. This insures a continuous definition of the effective behavior over the whole $(\boldsymbol{k}, \omega)$ space. However, to one microscopic free wave will correspond an infinity of effective ones, one for each limit process or, as remarked earlier, one for each Fourier component. Therefore, the effective behavior, by superposition of Fourier components, will be able to reproduce exactly the microscopic free wave, which is physical, but also an infinity of other non-physical free waves.

2. Definition (2.3) can be extended thanks to the long-wavelength-component interpretation (2.9). However, this is not without restricting wavenumbers to the first Brillouin zone $T^{*}$ as previously discussed. Accordingly, the effective behavior will have a meaning only for modes $(\boldsymbol{k}, \omega)$ such that $\boldsymbol{k} \in T^{*}$.

3. Finally, the effective wavenumber $\boldsymbol{k}+\boldsymbol{\xi}$ can be chosen so that $\boldsymbol{U}$ is the best approximation of $\boldsymbol{u}$ in some sense.

Let us discuss the last choice in more details. The quality of the approximation $\boldsymbol{U}$ of $\boldsymbol{u}$ depends on the choice of the effective wavenumber $\boldsymbol{k}$ which represents the class $\boldsymbol{k} \bmod \mathscr{R}^{*}$. To assess this quality, an error function must be defined. Srivastava and Nemat-Nasser (2014) have recently studied this question and defined the error as the normalized reflected energy over an interface separating the effective medium from the original one and gave results based on the study of some particular layered composites. Here, we define the error by comparing the order of magnitudes of two terms constituting the microscopic elastic energy. Though our approach is more general, it only yields a necessary condition for this error to be small hereafter called third homogenizability condition.

As a matter of fact, calling $\boldsymbol{k}$ the chosen effective wavenumber, for $\boldsymbol{U}$ to be a physically good approximation of $\boldsymbol{u}$, components $\left\{\tilde{\boldsymbol{u}}_{\boldsymbol{\xi}}, \boldsymbol{\xi} \neq \mathbf{0}\right\}$ should be small, in the strain-energy sense for example, with respect to the component $\tilde{\boldsymbol{u}}_{\mathbf{0}}=\langle\tilde{\boldsymbol{u}}\rangle$. This can be written as

$$
\left\langle\left[\boldsymbol{C}:\left(\boldsymbol{\nabla} \otimes^{s} \tilde{\boldsymbol{u}}\right)\right]:\left(\boldsymbol{\nabla} \otimes^{s} \tilde{\boldsymbol{u}}^{*}\right)\right\rangle \ll\left\langle\left[\boldsymbol{C}:\left(i \boldsymbol{k} \otimes^{s} \tilde{\boldsymbol{u}}\right)\right]:\left(i \boldsymbol{k} \otimes^{s} \tilde{\boldsymbol{u}}\right)^{*}\right\rangle .
$$

In fact, the strain field $\varepsilon$ has the expression

$$
\boldsymbol{\varepsilon}=\boldsymbol{\nabla} \otimes^{s} \boldsymbol{u}=\boldsymbol{\nabla} \otimes^{s}\left(\tilde{\boldsymbol{u}} e^{i \boldsymbol{k} \cdot \boldsymbol{x}}\right)=\left(\boldsymbol{\nabla} \otimes^{s} \tilde{\boldsymbol{u}}\right) e^{i \boldsymbol{k} \cdot \boldsymbol{x}}+\left(i \boldsymbol{k} \otimes^{s} \tilde{\boldsymbol{u}}\right) e^{i \boldsymbol{k} \cdot \boldsymbol{x}} \equiv \tilde{\boldsymbol{\varepsilon}} e^{i \boldsymbol{k} \cdot \boldsymbol{x}} .
$$

Condition (2.11) implies that the microscopic variations due to $\tilde{\boldsymbol{u}}$ are small with respect to the variations due to the effective wavenumber $\boldsymbol{k}$. To exploit this hypothesis, we invoke the virtual work principle (recall that $\boldsymbol{f}=\mathbf{0}$ ):

$$
\omega^{2}\left\langle\rho \boldsymbol{u} \cdot \boldsymbol{u}^{*}\right\rangle=\left\langle(\boldsymbol{C}: \boldsymbol{\varepsilon}): \boldsymbol{\varepsilon}^{*}\right\rangle .
$$


The triangle inequality has the consequence that

$$
\omega^{2}\left\langle\rho \boldsymbol{u} \cdot \boldsymbol{u}^{*}\right\rangle \leq\left(\sqrt{\left\langle\left[\boldsymbol{C}:\left(\boldsymbol{\nabla} \otimes^{s} \tilde{\boldsymbol{u}}\right)\right]:\left(\boldsymbol{\nabla} \otimes^{s} \tilde{\boldsymbol{u}}^{*}\right)\right\rangle}+\sqrt{\left\langle\left[\boldsymbol{C}:\left(i \boldsymbol{k} \otimes^{s} \boldsymbol{u}\right)\right]:\left(i \boldsymbol{k} \otimes^{s} \boldsymbol{u}\right)^{*}\right\rangle}\right)^{2}
$$

Hence, the condition (2.11) yields

$$
\omega^{2}\left\langle\rho \boldsymbol{u} \cdot \boldsymbol{u}^{*}\right\rangle \lesssim\left\langle\left[\boldsymbol{C}:\left(i \boldsymbol{k} \otimes^{s} \boldsymbol{u}\right)\right]:\left(i \boldsymbol{k} \otimes^{s} \boldsymbol{u}\right)^{*}\right\rangle
$$

where the relation $\lesssim$ is defined as "smaller than or almost equal to". Consequently, we have

$$
\omega^{2} \lesssim \frac{\left\langle\left[\boldsymbol{C}:\left(i \boldsymbol{k} \otimes^{s} \boldsymbol{u}\right)\right]:\left(i \boldsymbol{k} \otimes^{s} \boldsymbol{u}\right)^{*}\right\rangle}{\left\langle\rho \boldsymbol{u} \cdot \boldsymbol{u}^{*}\right\rangle} .
$$

Next, we compare the $\boldsymbol{C}$-norm and the $\rho$-norm. In this regard, we have

$$
\begin{aligned}
& \left\langle\left[\boldsymbol{C}:\left(i \boldsymbol{k} \otimes^{s} \boldsymbol{u}\right)\right]:\left(i \boldsymbol{k} \otimes^{s} \boldsymbol{u}\right)^{*}\right\rangle=\left\langle\rho\left[\frac{\boldsymbol{C}}{\rho}:\left(i \boldsymbol{k} \otimes^{s} \boldsymbol{u}\right)\right]:\left(i \boldsymbol{k} \otimes^{s} \boldsymbol{u}\right)^{*}\right\rangle \\
& \leq\left\langle\rho\left(i \boldsymbol{k} \otimes^{s} \boldsymbol{u}\right):\left(i \boldsymbol{k} \otimes^{s} \boldsymbol{u}\right)^{*}\right\rangle \max _{\substack{\boldsymbol{x} \in T \\
I=1 \ldots 6}}\left(\frac{c_{I}(\boldsymbol{x})}{\rho(\boldsymbol{x})}\right) \leq(\boldsymbol{k} \cdot \boldsymbol{k})\left\langle\rho \boldsymbol{u} \cdot \boldsymbol{u}^{*}\right\rangle \max _{\substack{\boldsymbol{x} \in T \\
I=1 \ldots 6}}\left(\frac{c_{I}(\boldsymbol{x})}{\rho(\boldsymbol{x})}\right)
\end{aligned}
$$

where the $\left(c_{I}\right)_{I=1 \ldots 6}$ are the eigenvalues of the elasticity tensor $\boldsymbol{C}$. It can then be concluded that

$$
\omega^{2} \lesssim \max _{\substack{\boldsymbol{x} \in T \\ I=1 \ldots 6}}\left(\frac{c_{I}(\boldsymbol{x})}{\rho(\boldsymbol{x})}\right)\|\boldsymbol{k}\|^{2} .
$$

This third homogenizability condition says that for high frequencies, the elastic energy is carried by short wavelengths (see figures 9 and 10 for examples that are to be introduced later). It gives accordingly a necessary condition on the optimal choice of $\boldsymbol{k}$ which can yield a relatively small error between $\boldsymbol{U}$ and $\boldsymbol{u}$. The higher the frequency is, the shorter the effective wavelength must be. High frequencies tend therefore to violate the second homogenizability condition for free waves.

Requiring both conditions (2.10) and (2.12) yields an upper bound for the set of homogenizable frequencies $\omega$ :

$$
\omega^{2} \lesssim \max _{\substack{\boldsymbol{x} \in T \\ I=1 \ldots 6}}\left(\frac{c_{I}(\boldsymbol{x})}{\rho(\boldsymbol{x})}\right) \max _{\boldsymbol{k} \in T^{*}}\|\boldsymbol{k}\|^{2} \equiv \max _{\substack{\boldsymbol{x} \in T \\ I=1 \ldots 6}}\left(\frac{c_{I}(\boldsymbol{x})}{\rho(\boldsymbol{x})}\right) \frac{\pi^{2}}{4 \ell^{2}},
$$

where $\ell$ is a microstructural length depending only on the geometry of $\mathscr{R}$. The homogenizable frequency-domain can contain the acoustic branches of the dispersion curve and, depending on the actual microstructure of $\Omega$, some of the optical branches. This result is in agreement with and generalizes that of Srivastava and NematNasser (2014). The cited authors proved that, for particular layered composites, 
the acoustic branch was homogenizable (in their particular sense presented above) and so is the first optical branch for composites with internal resonances.

Finally, condition (2.13) can be seen as a relaxed scale separation condition in comparison with the following classical one

$$
\omega^{2} \ll \min _{I=1 \ldots 6}\left(\frac{c_{I}^{m}}{\rho^{m}}\right) \frac{\pi^{2}}{4 \ell_{m}^{2}},
$$

for all phases $m$, where $\ell_{m}$ means the "radius" of phase $m$.

\subsection{Formal solutions and effective relation}

In this subsection, the methodology developed by Willis (2011) to derive effective constitutive laws is revisited in such a way that the main aspects for periodic media are specified and clarified. In fact, we present a purely spatial ${ }^{7}$ counterpart of said methodology.

\subsubsection{Preliminary discussion}

In deriving the effective constitutive relation, the solution of (2.2) needs to be expressed first in terms of the applied loadings (representation step) and then in terms of the effective strain and velocity (localization step). Formally, in the localization step, we write

$$
\tilde{\boldsymbol{u}}=\langle\tilde{\boldsymbol{u}}\rangle+\boldsymbol{A}:\langle\tilde{\varepsilon}\rangle+\boldsymbol{B} \cdot\langle\tilde{\boldsymbol{v}}\rangle
$$

where $\boldsymbol{A}$ and $\boldsymbol{B}$ are two localization tensor fields to be determined. However, $\langle\tilde{\boldsymbol{\varepsilon}}\rangle$ and $\langle\tilde{\boldsymbol{v}}\rangle$ are not algebraically independent since:

$$
i \omega\langle\tilde{\boldsymbol{\varepsilon}}\rangle=i \omega\left(\left\langle\boldsymbol{\nabla} \otimes^{s} \tilde{\boldsymbol{u}}\right\rangle+i \boldsymbol{k} \otimes^{s}\langle\tilde{\boldsymbol{u}}\rangle\right)=i \boldsymbol{k} \otimes^{s}\langle\tilde{\boldsymbol{v}}\rangle,
$$

where $\boldsymbol{\nabla} \otimes{ }^{s} \tilde{\boldsymbol{u}}$ has zero average given the periodic boundary conditions. This will result in an ambiguity in the definition of tensors $\boldsymbol{A}$ and $\boldsymbol{B}$.

The foregoing fact that velocity and strain are dependent is unusual. Indeed, for a local medium, the strain tensor $\boldsymbol{\varepsilon}\left(\boldsymbol{x}_{\circ}\right)$ and the velocity vector $\boldsymbol{v}\left(\boldsymbol{x}_{\circ}\right)$ at a given point $\boldsymbol{x}_{\circ}$ are independent of each other and they are also independent of the displacement $\boldsymbol{u}\left(\boldsymbol{x}_{\circ}\right)$ at the same point. However, for a nonlocal medium, the variables are some fields over all the medium. For example, the strain tensor and velocity fields are not independent since the time derivative of the former is equal to the symmetric part of the space gradient of the latter. The effective behavior of the periodic medium once homogenized is, a priori, nonlocal and characterized by a relation between some $(\boldsymbol{x}, t)$-dependent effective fields over $\Omega$. In contrast,

\footnotetext{
${ }^{7}$ This is: without referring to random descriptions.
} 
some numerical homogenization schemes seek, at isolated macroscopic points, for an effective behavior which links total averages of the microscopic fields. Such schemes do not seem to have to deal with $\boldsymbol{E}-\boldsymbol{V}$ dependency, because they explicitly presume locality through the use of spatial Taylor expansions and, thus, leave no place for a nonlocal behavior (see (Pham et al., 2013)).

One way to solve the ambiguity in definition of tensors $\boldsymbol{A}$ and $\boldsymbol{B}$ is to prohibit "abusive" time integration and derivation (i.e., division and multiplication by $i \omega$ ). To this end, let $\gamma(\boldsymbol{x})=\tilde{\boldsymbol{\gamma}} e^{i \boldsymbol{k} \cdot \boldsymbol{x}}$ be a plane-wave eigenstrain field over $\Omega$ so that the stress-strain relationship becomes

$$
\sigma=C:(\varepsilon-\gamma)
$$

Now, since $\boldsymbol{\gamma}$ do not derive from the displacement field $\boldsymbol{u}$ and is therefore independent of $\boldsymbol{v}$, by keeping track of $(\varepsilon-\gamma)$ as a whole, no confusion between the strain and velocity components is possible. Correspondingly, equation (2.2) takes the form

$$
(\boldsymbol{\nabla}+i \boldsymbol{k}) \cdot\left\{\boldsymbol{C}:\left[(\boldsymbol{\nabla}+i \boldsymbol{k}) \otimes^{s} \tilde{\boldsymbol{u}}-\tilde{\boldsymbol{\gamma}}\right]\right\}+\tilde{\boldsymbol{f}}=-\omega^{2} \rho \tilde{\boldsymbol{u}}
$$

where $\tilde{\gamma}$ can be viewed as a generalized loading. The use of eigenstrain in (Willis, 2011) was inspired by the numerical scheme of Fietz and Shvets (2010).

We underline the fact that non-uniqueness is not due to a particular choice of boundary conditions, even though the dependency takes a simple algebraic form in this periodic case. This is mentioned explicitly in the more general study (Willis, 2011). Incidentally, eigenstrain $\boldsymbol{\gamma}$ can be seen to play the dual role of body force $\boldsymbol{f}$. If $\boldsymbol{f}$ was not taken into account, stress $\boldsymbol{\Sigma}$ and momentum $\boldsymbol{P}$ would have been dependent through the effective motion equation:

$$
i \boldsymbol{k} \cdot \boldsymbol{\Sigma}=i \omega \boldsymbol{P}
$$

which is equivalent to restraining our attention to free wave solutions. The bottom line is that different loadings will allow to investigate more general aspects of the effective behavior. We also refer to the discussion presented in (Willis, 2012) for other interesting aspects of non-uniqueness.

Here, we have adopted the choice of localizing $\boldsymbol{u}$ with respect to $\boldsymbol{E}-\boldsymbol{\gamma}$ and $\boldsymbol{V}$. Other legitimate choices exist. For example, we could have renounced the use of $\gamma$ and localized $\boldsymbol{u}$ directly with respect to $\boldsymbol{U}$ (as sketched in (Willis, 2009)). Such choices will lead to other forms of the constitutive equations, which are, however, compatible in the sense that they give rise to the same motion equation in terms of $\boldsymbol{U}$.

\subsubsection{Representation equation}

We define component $g_{i j}(\boldsymbol{x}, \boldsymbol{y})=\psi_{i}(\boldsymbol{x})$ of the Green tensor $\boldsymbol{g}$ as the $i^{\text {th }}$ component, at $\boldsymbol{x}$, of the solution of the following equation, where $\boldsymbol{y}$ and $j$ are merely 
parameters, under periodic boundary conditions imposed on $T$ :

$$
(\boldsymbol{\nabla}+i \boldsymbol{k}) \cdot\left\{\boldsymbol{C}:\left[(\boldsymbol{\nabla}+i \boldsymbol{k}) \otimes^{s} \boldsymbol{\psi}\right]\right\}+|T| \delta_{\boldsymbol{y}} \boldsymbol{e}_{j}=-\omega^{2} \rho \boldsymbol{\psi}
$$

where $\boldsymbol{e}_{j}$ is the $j^{\text {th }}$ vector of an orthonormal basis of the space $\mathscr{E}$ and $\delta_{\boldsymbol{y}}$ is the Dirac function. Letting $\boldsymbol{\psi}$ and $\tilde{\boldsymbol{u}}$ be kinematically admissible displacements for equations (2.14) and (2.15), respectively, the virtual work principle yields

$$
\begin{aligned}
\left\langle\tilde{\boldsymbol{f}} \cdot \boldsymbol{\psi}^{*}\right\rangle & =\left\langle[\boldsymbol{C}:(\tilde{\boldsymbol{\varepsilon}}-\tilde{\gamma})]:\left[(\boldsymbol{\nabla}+i \boldsymbol{k}) \otimes^{s} \boldsymbol{\psi}\right]^{*}-\omega^{2} \rho \tilde{\boldsymbol{u}} \cdot \boldsymbol{\psi}^{*}\right\rangle, \\
\left\langle|T| \delta_{\boldsymbol{y}} \boldsymbol{e}_{j} \cdot \tilde{\boldsymbol{u}}^{*}\right\rangle & =\left\langle\left\{\boldsymbol{C}:\left[(\boldsymbol{\nabla}+i \boldsymbol{k}) \otimes^{s} \boldsymbol{\psi}\right]\right\}: \tilde{\boldsymbol{\varepsilon}}^{*}-\omega^{2} \rho \boldsymbol{\psi} \cdot \tilde{\boldsymbol{u}}^{*}\right\rangle .
\end{aligned}
$$

Since $\boldsymbol{C}$ is a real symmetric tensor and $\rho$ is a real scalar, the difference between the first of the preceding two equations and the complex conjugate of the second leads to

$$
-\left\langle|T| \delta_{\boldsymbol{y}} \boldsymbol{e}_{j} \cdot \tilde{\boldsymbol{u}}\right\rangle+\left\langle\tilde{\boldsymbol{f}} \cdot \boldsymbol{\psi}^{*}\right\rangle=-\left\langle(\boldsymbol{C}: \tilde{\boldsymbol{\gamma}}):\left[(\boldsymbol{\nabla}+i \boldsymbol{k}) \otimes^{s} \boldsymbol{\psi}\right]^{*}\right\rangle .
$$

This is the sought representation equation in a rather implicit form.

Equation (2.16) is valid for all triplets $(\tilde{\boldsymbol{u}}, \tilde{\boldsymbol{f}}, \tilde{\gamma})$ and in particular for $\tilde{\boldsymbol{\gamma}}=\mathbf{0}$, $\tilde{\boldsymbol{f}}=|T| \delta_{\boldsymbol{x}} \boldsymbol{e}_{i}$ and, accordingly, $\tilde{u}_{j}(\boldsymbol{y})=g_{j i}(\boldsymbol{y}, \boldsymbol{x})$, which yields ${ }^{8}$

$$
g_{i j}(\boldsymbol{x}, \boldsymbol{y})=g_{j i}(\boldsymbol{y}, \boldsymbol{x})^{*} .
$$

With the aid of this symmetry property of $\boldsymbol{g}$, let us rewrite the representation equation (2.16) in a more explicit way:

$$
|T| \tilde{\boldsymbol{u}}(\boldsymbol{y})=\int_{T} \boldsymbol{g}(\boldsymbol{y}, \boldsymbol{x}) \cdot \tilde{\boldsymbol{f}} \mathrm{d} \boldsymbol{x}+\int_{T}\left[\boldsymbol{g}(\boldsymbol{y}, \boldsymbol{x}) \otimes^{s}\left(\nabla_{\boldsymbol{x}}-i \boldsymbol{k}\right)\right]:[\boldsymbol{C}(\boldsymbol{x}): \tilde{\gamma}] \mathrm{d} \boldsymbol{x}
$$

where $\boldsymbol{\nabla}_{\boldsymbol{x}}$ is the usual differential operator applied to the $\boldsymbol{x}$ variable. The operator $\boldsymbol{\nabla}_{\boldsymbol{y}}$ will be similarly understood. In addition, use will be made of the volume average operators $\langle\cdot\rangle_{\boldsymbol{x}},\langle\cdot\rangle_{\boldsymbol{y}}$ and $\langle\langle\cdot\rangle\rangle$ with respect to $\boldsymbol{x}, \boldsymbol{y}$ and both $\boldsymbol{x}$ and $\boldsymbol{y}$, respectively. However, no distinction is indicated when confusion is impossible, i.e., for fields of one variable. Unless otherwise specified, the symbol $\boldsymbol{g}$ stands for $\boldsymbol{g}(\boldsymbol{y}, \boldsymbol{x})$ hereafter. A last notation convention is that all fields written on the right of the operator $\boldsymbol{g}$ are $\boldsymbol{x}$-dependent while all those on the left of $\boldsymbol{g}$ are $\boldsymbol{y}$-dependent. Finally, equation (2.18) can be written in the following compact form ${ }^{9}$ :

$$
\tilde{\boldsymbol{u}}=\langle\boldsymbol{g}\rangle_{\boldsymbol{x}} \cdot \tilde{\boldsymbol{f}}+\left\langle\left[\boldsymbol{g} \otimes^{s}\left(\nabla_{x}-i \boldsymbol{k}\right)\right]: \boldsymbol{C}\right\rangle_{\boldsymbol{x}}: \tilde{\gamma}
$$

where $\tilde{\boldsymbol{f}}$ and $\tilde{\boldsymbol{\gamma}}$ are homogeneous.

\footnotetext{
${ }^{8}$ For a thorough study of fundamental solutions $\boldsymbol{g}$, one may refer to (Bonnet, 1995).

${ }^{9}$ This is an equality between vector fields of the variable $\boldsymbol{y}$. The operator $\boldsymbol{g}$ is to be seen as a second order tensor.
} 


\subsubsection{Localization}

Aiming to localize $\tilde{\boldsymbol{u}}$ with respect to $\langle\tilde{\boldsymbol{v}}\rangle$ and to $\langle\tilde{\boldsymbol{\varepsilon}}-\tilde{\boldsymbol{\gamma}}\rangle$, we can re-write $\tilde{\boldsymbol{\gamma}}$ as $-\langle\tilde{\varepsilon}-\tilde{\gamma}\rangle+\langle\tilde{\varepsilon}\rangle$ in equation (2.19) which becomes

$$
\begin{aligned}
& \tilde{\boldsymbol{u}}=\langle\boldsymbol{g}\rangle_{\boldsymbol{x}} \cdot \tilde{\boldsymbol{f}}-\left\langle\left[\boldsymbol{g} \otimes^{s}\left(\nabla_{x}-i \boldsymbol{k}\right)\right]: \boldsymbol{C}\right\rangle_{\boldsymbol{x}}:\langle\tilde{\boldsymbol{\varepsilon}}-\tilde{\gamma}\rangle \\
& +\left\langle\left[\boldsymbol{g} \otimes^{s}\left(\boldsymbol{\nabla}_{\boldsymbol{x}}-i \boldsymbol{k}\right)\right]: \boldsymbol{C}\right\rangle_{\boldsymbol{x}}:\langle\tilde{\boldsymbol{\varepsilon}}\rangle .
\end{aligned}
$$

The third term in the right-side member of this equation can be further written as

$$
\begin{aligned}
\left\langle\left[\boldsymbol{g} \otimes^{s}\left(\boldsymbol{\nabla}_{\boldsymbol{x}}-i \boldsymbol{k}\right)\right]: \boldsymbol{C}\right\rangle_{\boldsymbol{x}}:\langle\tilde{\boldsymbol{\varepsilon}}\rangle & =\left\langle\left[\boldsymbol{g} \otimes^{s}\left(\boldsymbol{\nabla}_{\boldsymbol{x}}-i \boldsymbol{k}\right)\right]: \boldsymbol{C}\right\rangle_{\boldsymbol{x}}:\left(i \boldsymbol{k} \otimes^{s}\langle\tilde{\boldsymbol{u}}\rangle\right) \\
& =\left\{\left\langle\left[\boldsymbol{g} \otimes^{s}\left(\boldsymbol{\nabla}_{\boldsymbol{x}}-i \boldsymbol{k}\right)\right]: \boldsymbol{C}\right\rangle_{\boldsymbol{x}} \cdot i \boldsymbol{k}\right\} \cdot\langle\tilde{\boldsymbol{u}}\rangle .
\end{aligned}
$$

At the time, the motion equation (2.15) of $\boldsymbol{g}$ averaged with respect to $\boldsymbol{x}$ gives

$$
i \boldsymbol{k} \cdot\left\langle\boldsymbol{C}(\boldsymbol{x}):\left[\left(\boldsymbol{\nabla}_{\boldsymbol{x}}+i \boldsymbol{k}\right) \otimes^{s} \boldsymbol{g}(\boldsymbol{x}, \boldsymbol{y})\right]\right\rangle_{\boldsymbol{x}}+\boldsymbol{I}=-\omega^{2}\langle\rho(\boldsymbol{x}) \boldsymbol{g}(\boldsymbol{x}, \boldsymbol{y})\rangle_{\boldsymbol{x}}
$$

where $\boldsymbol{I}$ is the second order identity tensor. Transposing the above equation, we obtain

$$
\left\langle\left[\boldsymbol{g}^{\top}(\boldsymbol{x}, \boldsymbol{y}) \otimes^{s}\left(\nabla_{\boldsymbol{x}}+i \boldsymbol{k}\right)\right]: \boldsymbol{C}(\boldsymbol{x})\right\rangle_{\boldsymbol{x}} \cdot i \boldsymbol{k}+\boldsymbol{I}=-\omega^{2}\left\langle\boldsymbol{g}^{\top}(\boldsymbol{x}, \boldsymbol{y}) \rho(\boldsymbol{x})\right\rangle_{\boldsymbol{x}},
$$

with the superscript $\left({ }^{\top}\right)$ standing for transposition. Accounting for the symmetry (2.17) of $\boldsymbol{g}$, it follows that

$$
\left\langle\left[\boldsymbol{g}^{*}(\boldsymbol{y}, \boldsymbol{x}) \otimes^{s}\left(\boldsymbol{\nabla}_{\boldsymbol{x}}+i \boldsymbol{k}\right)\right]: \boldsymbol{C}(\boldsymbol{x})\right\rangle_{\boldsymbol{x}} \cdot i \boldsymbol{k}+\boldsymbol{I}=-\omega^{2}\left\langle\boldsymbol{g}^{*}(\boldsymbol{y}, \boldsymbol{x}) \rho(\boldsymbol{x})\right\rangle_{\boldsymbol{x}},
$$

whose complex conjugate takes the form

$$
\left\langle\left[\boldsymbol{g}(\boldsymbol{y}, \boldsymbol{x}) \otimes^{s}\left(\nabla_{\boldsymbol{x}}-i \boldsymbol{k}\right)\right]: \boldsymbol{C}(\boldsymbol{x})\right\rangle_{\boldsymbol{x}} \cdot i \boldsymbol{k}=\boldsymbol{I}+\omega^{2}\langle\boldsymbol{g}(\boldsymbol{y}, \boldsymbol{x}) \rho(\boldsymbol{x})\rangle_{\boldsymbol{x}} .
$$

Combining (2.20), (2.21) and (2.22) delivers

$$
\tilde{\boldsymbol{u}}-\langle\tilde{\boldsymbol{u}}\rangle=\langle\boldsymbol{g}\rangle_{\boldsymbol{x}} \cdot \tilde{\boldsymbol{f}}-\left\langle\left[\boldsymbol{g} \otimes^{s}\left(\nabla_{\boldsymbol{x}}-i \boldsymbol{k}\right)\right]: \boldsymbol{C}\right\rangle_{\boldsymbol{x}}:\langle\tilde{\boldsymbol{\varepsilon}}-\tilde{\boldsymbol{\gamma}}\rangle+\omega^{2}\langle\boldsymbol{g} \rho\rangle_{\boldsymbol{x}} \cdot\langle\tilde{\boldsymbol{u}}\rangle .
$$

The volume average of this equation with respect to $\boldsymbol{y}$ results in an algebraic equation

$$
\mathbf{0}=\langle\langle\boldsymbol{g}\rangle\rangle \cdot \tilde{\boldsymbol{f}}-\left\langle\left\langle\left[\boldsymbol{g} \otimes^{s}\left(\nabla_{\boldsymbol{x}}-i \boldsymbol{k}\right)\right]: \boldsymbol{C}\right\rangle\right\rangle:\langle\tilde{\boldsymbol{\varepsilon}}-\tilde{\gamma}\rangle+\omega^{2}\langle\langle\boldsymbol{g} \rho\rangle\rangle \cdot\langle\tilde{\boldsymbol{u}}\rangle
$$

whose solution for $\tilde{\boldsymbol{f}}$ is given by

$$
\tilde{\boldsymbol{f}}=\langle\langle\boldsymbol{g}\rangle\rangle^{-1} \cdot\left\langle\left\langle\left[\boldsymbol{g} \otimes^{s}\left(\nabla_{\boldsymbol{x}}-i \boldsymbol{k}\right)\right]: \boldsymbol{C}\right\rangle\right\rangle:\langle\tilde{\boldsymbol{\varepsilon}}-\tilde{\gamma}\rangle-\omega^{2}\langle\langle\boldsymbol{g}\rangle\rangle^{-1} \cdot\langle\langle\boldsymbol{g} \rho\rangle\rangle \cdot\langle\tilde{\boldsymbol{u}}\rangle
$$

provided $\operatorname{det}\langle\langle\boldsymbol{g}\rangle\rangle \neq 0$. Introducing (2.24) into (2.23), we finally obtain

$$
\tilde{\boldsymbol{u}}(\boldsymbol{y})-\langle\tilde{\boldsymbol{u}}\rangle=\boldsymbol{A}(\boldsymbol{y}):\langle\tilde{\varepsilon}-\tilde{\gamma}\rangle+\boldsymbol{B}(\boldsymbol{y}) \cdot\langle\tilde{\boldsymbol{v}}\rangle,
$$


with

$$
\begin{aligned}
& \boldsymbol{A}=\langle\boldsymbol{g}\rangle_{\boldsymbol{x}} \cdot\langle\langle\boldsymbol{g}\rangle\rangle^{-1} \cdot\left\langle\left\langle\left[\boldsymbol{g} \otimes^{s}\left(\nabla_{\boldsymbol{x}}-i \boldsymbol{k}\right)\right]: \boldsymbol{C}\right\rangle\right\rangle-\left\langle\left[\boldsymbol{g} \otimes^{s}\left(\nabla_{\boldsymbol{x}}-i \boldsymbol{k}\right)\right]: \boldsymbol{C}\right\rangle_{\boldsymbol{x}}, \\
& \boldsymbol{B}=i \omega\langle\boldsymbol{g}\rangle_{\boldsymbol{x}} \cdot\langle\langle\boldsymbol{g}\rangle\rangle^{-1} \cdot\langle\langle\boldsymbol{g} \rho\rangle\rangle-i \omega\langle\boldsymbol{g} \rho\rangle_{\boldsymbol{x}} .
\end{aligned}
$$

Then, it is immediate that

$$
\begin{aligned}
\tilde{\boldsymbol{\sigma}} & =\left\{\boldsymbol{C}+\boldsymbol{C}:\left[\left(\boldsymbol{\nabla}_{\boldsymbol{y}}+i \boldsymbol{k}\right) \otimes^{s} \boldsymbol{A}\right]\right\}:\langle\tilde{\boldsymbol{\varepsilon}}-\tilde{\gamma}\rangle+\left\{\boldsymbol{C}:\left[\left(\nabla_{\boldsymbol{y}}+i \boldsymbol{k}\right) \otimes^{s} \boldsymbol{B}\right]\right\} \cdot\langle\tilde{\boldsymbol{v}}\rangle, \\
\tilde{\boldsymbol{p}} & =i \omega \rho \boldsymbol{A}:\langle\tilde{\varepsilon}-\tilde{\gamma}\rangle+(\rho \boldsymbol{I}+i \omega \rho \boldsymbol{B}) \cdot\langle\tilde{\boldsymbol{v}}\rangle .
\end{aligned}
$$

\subsubsection{Homogenization step}

With the expressions for $\tilde{\boldsymbol{\sigma}}$ and $\tilde{\boldsymbol{p}}$, from equation (2.26), the homogenization step can be accomplished easily by volume averaging. Indeed, using the definition (2.3) for $\boldsymbol{\Sigma}$ and $\boldsymbol{P}$, the effective constitutive law of a periodic medium is specified by

$$
\left[\begin{array}{c}
\boldsymbol{\Sigma} \\
\boldsymbol{P}
\end{array}\right]=\left[\begin{array}{ll}
\boldsymbol{C}^{e} & \boldsymbol{S}^{1} \\
\boldsymbol{S}^{2} & \boldsymbol{\rho}^{e}
\end{array}\right]_{k, \omega}\left[\begin{array}{c}
\boldsymbol{E}-\boldsymbol{\gamma} \\
\boldsymbol{V}
\end{array}\right]
$$

where the fourth-order effective stiffness tensor $\boldsymbol{C}^{e}$, the second-order effective mass tensor $\boldsymbol{\rho}^{e}$ and the third-order coupling tensors $\boldsymbol{S}^{1}$ and $\boldsymbol{S}^{2}$ depend on $(\boldsymbol{k}, \omega)$ and have the expressions

$$
\begin{aligned}
& \boldsymbol{C}^{e}=\langle\boldsymbol{C}\rangle+\left\langle\boldsymbol{C}:\left[\left(\boldsymbol{\nabla}_{\boldsymbol{y}}+i \boldsymbol{k}\right) \otimes^{s} \boldsymbol{A}\right]\right\rangle, \quad \boldsymbol{\rho}^{e}=\langle\rho\rangle \boldsymbol{I}+i \omega\langle\rho \boldsymbol{B}\rangle, \\
& \boldsymbol{S}^{1}=\left\langle\boldsymbol{C}:\left[\left(\boldsymbol{\nabla}_{\boldsymbol{y}}+i \boldsymbol{k}\right) \otimes^{s} \boldsymbol{B}\right]\right\rangle \quad \text { and } \quad \boldsymbol{S}^{2}=i \omega\langle\rho \boldsymbol{A}\rangle .
\end{aligned}
$$

Accounting for the formulae (2.25) for $\boldsymbol{A}$ and $\boldsymbol{B}$, the constitutive tensors can further be specified as follows (an equivalent form of equation (3.19) of (Willis, 2011)):

$$
\begin{aligned}
\boldsymbol{C}^{e}=\langle\boldsymbol{C}\rangle+\left\langle\left\langle\boldsymbol{C}:\left[\left(\nabla_{\boldsymbol{y}}+i \boldsymbol{k}\right) \otimes^{s} \boldsymbol{g}\right]\right\rangle\right\rangle \cdot\langle\langle\boldsymbol{g}\rangle\rangle^{-1} \cdot\left\langle\left\langle\left[\boldsymbol{g} \otimes^{s}\left(\nabla_{\boldsymbol{x}}-i \boldsymbol{k}\right)\right]: \boldsymbol{C}\right\rangle\right\rangle \\
\quad-\left\langle\left\langle\boldsymbol{C}:\left[\left(\nabla_{\boldsymbol{y}}+i \boldsymbol{k}\right) \otimes^{s} \boldsymbol{g} \otimes^{s}\left(\nabla_{\boldsymbol{x}}-i \boldsymbol{k}\right)\right]: \boldsymbol{C}\right\rangle\right\rangle, \\
\boldsymbol{\rho}^{e}=\langle\rho\rangle \boldsymbol{I}-\omega^{2}\langle\langle\rho \boldsymbol{g}\rangle\rangle \cdot\langle\langle\boldsymbol{g}\rangle\rangle^{-1} \cdot\langle\langle\boldsymbol{g} \rho\rangle\rangle+\omega^{2}\langle\langle\rho \boldsymbol{g} \rho\rangle\rangle, \\
\boldsymbol{S}^{1}=i \omega\left\langle\left\langle\boldsymbol{C}:\left[\left(\boldsymbol{\nabla}_{\boldsymbol{y}}+i \boldsymbol{k}\right) \otimes^{s} \boldsymbol{g}\right]\right\rangle\right\rangle \cdot\langle\langle\boldsymbol{g}\rangle\rangle^{-1} \cdot\langle\langle\boldsymbol{g} \rho\rangle\rangle-i \omega\left\langle\left\langle\boldsymbol{C}:\left[\left(\boldsymbol{\nabla}_{\boldsymbol{y}}+i \boldsymbol{k}\right) \otimes^{s} \boldsymbol{g}\right] \rho\right\rangle\right\rangle, \\
\boldsymbol{S}^{2}=i \omega\langle\langle\rho \boldsymbol{g}\rangle\rangle \cdot\langle\langle\boldsymbol{g}\rangle\rangle^{-1} \cdot\left\langle\left\langle\left\langle\boldsymbol{g} \otimes^{s}\left(\nabla_{\boldsymbol{x}}-i \boldsymbol{k}\right)\right]: \boldsymbol{C}\right\rangle\right\rangle-i \omega\left\langle\left\langle\rho\left[\boldsymbol{g} \otimes^{s}\left(\nabla_{\boldsymbol{x}}-i \boldsymbol{k}\right)\right]: \boldsymbol{C}\right\rangle\right\rangle .
\end{aligned}
$$

To use the constitutive law (2.27) in the real space and time domains, let us, for simplicity, set $\boldsymbol{\gamma}$ to zero and let $\boldsymbol{E}(\boldsymbol{x}, t)$ and $\boldsymbol{V}(\boldsymbol{x}, t)$ be two arbitrary (not necessarily plane waves) strain and velocity effective fields. Those fields can be written as a sum of harmonic plane waves indexed by $(\boldsymbol{k}, \omega)$. For example:

$$
\boldsymbol{E}(\boldsymbol{x}, t)=\int \boldsymbol{E}_{\boldsymbol{k}, \omega} e^{i(\boldsymbol{k} \cdot \boldsymbol{x}+\omega t)} \mathrm{d} \boldsymbol{k} \mathrm{d} \omega .
$$


The effective stress and momentum fields $(\boldsymbol{\Sigma}(\boldsymbol{x}, t), \boldsymbol{P}(\boldsymbol{x}, t))$ admit similar expansions. According to $(2.27)$, for all $(\boldsymbol{k}, \omega)$, the stress response is given by

$$
\Sigma_{k, \omega}=C_{k, \omega}^{e}: E_{k, \omega}+S_{k, \omega}^{1} \cdot V_{k, \omega}
$$

Application of the superposition principle yields

$$
\begin{aligned}
& \boldsymbol{\Sigma}(\boldsymbol{x}, t)=\boldsymbol{C}^{e}(\boldsymbol{x}, t)^{*}: \boldsymbol{E}(\boldsymbol{x}, t)+\boldsymbol{S}^{1}(\boldsymbol{x}, t)^{*} \cdot \boldsymbol{V}(\boldsymbol{x}, t) \\
& \quad=\frac{1}{(2 \pi)^{4}} \int\left(\boldsymbol{C}^{e}\left(\boldsymbol{x}-\boldsymbol{x}^{\prime}, t-t^{\prime}\right): \boldsymbol{E}\left(\boldsymbol{x}^{\prime}, t^{\prime}\right)+\boldsymbol{S}^{1}\left(\boldsymbol{x}-\boldsymbol{x}^{\prime}, t-t^{\prime}\right) \cdot \boldsymbol{V}\left(\boldsymbol{x}^{\prime}, t^{\prime}\right)\right) \mathrm{d} \boldsymbol{x}^{\prime} \mathrm{d} t^{\prime}
\end{aligned}
$$

where $\boldsymbol{C}^{e}(\boldsymbol{x}, t)$ and $\boldsymbol{S}^{1}(\boldsymbol{x}, t)$ are the inverse Fourier transforms of $\boldsymbol{C}_{\boldsymbol{k}, \omega}$ and $\boldsymbol{S}_{\boldsymbol{k}, \omega}^{1}$ while $\left({ }^{*}\right)$ and $\left(^{*}\right)$ are space-time convolution operations. The effective constitutive behavior of a periodic medium homogenized within the framework of elastodynamics appear then to be nonlocal both in space and time.

Finally, the effective constitutive equation (2.27) is clearly independent of the body force $\boldsymbol{f}$ and therefore is also independent of the prescribed initial conditions (see footnote 4 ).

\subsection{Reinterpreting the effective behavior: back to the homogenizability conditions}

The effective behavior has been derived with the help of plane-wave body forces. We have already discussed the effect of combining plane waves when presenting a generalized version of Hill-Mandel lemma. It was observed that combining body forces can lead to an error term. Here, we present the effect of such combinations on the effective behavior.

As can be seen from equation (2.7), only plane-wave body forces of wavenumbers separated by a reciprocal lattice vector can interact with one another. A general body force whose all wavenumbers are separated from each other by reciprocal lattice vectors is simply a Bloch wave:

$$
\boldsymbol{f}(\boldsymbol{x})=\tilde{\boldsymbol{f}}(\boldsymbol{x}) e^{i \boldsymbol{k} \cdot \boldsymbol{x}}=\left(\sum_{\boldsymbol{\xi} \in \mathscr{R}^{*}} \tilde{\boldsymbol{f}}_{\boldsymbol{\xi}} e^{i \boldsymbol{\xi} \cdot \boldsymbol{x}}\right) e^{i \boldsymbol{k} \cdot \boldsymbol{x}} .
$$

The microscopic displacement field has a similar expression:

$$
\boldsymbol{u}(\boldsymbol{x})=\tilde{\boldsymbol{u}}(\boldsymbol{x}) e^{i \boldsymbol{k} \cdot \boldsymbol{x}}=\left(\sum_{\boldsymbol{\xi} \in \mathscr{R}^{*}} \tilde{\boldsymbol{u}}_{\boldsymbol{\xi}} e^{i \boldsymbol{\xi} \cdot \boldsymbol{x}}\right) e^{i \boldsymbol{k} \cdot \boldsymbol{x}}
$$

and the representation equation (2.19) still hold and reads

$$
\tilde{\boldsymbol{u}}(\boldsymbol{y})=\langle\boldsymbol{g}(\boldsymbol{y}, \boldsymbol{x}) \cdot \tilde{\boldsymbol{f}}(\boldsymbol{x})\rangle_{\boldsymbol{x}}
$$


Using Fourier series expansion, the above equation becomes

$$
\forall \boldsymbol{\zeta} \in \mathscr{R}^{*}, \quad \tilde{\boldsymbol{u}}_{\zeta}=\sum_{\boldsymbol{\xi} \in \mathscr{R}^{*}}\left\langle\left\langle e^{i \zeta \cdot y} \boldsymbol{g}(\boldsymbol{y}, \boldsymbol{x}) e^{i \boldsymbol{\xi} \cdot \boldsymbol{x}}\right\rangle\right\rangle \cdot \tilde{\boldsymbol{f}}_{\boldsymbol{\xi}}
$$

The above equations solve the unit cell problem (2.2) Fourier-series expanded:

$$
\forall \boldsymbol{\zeta} \in \mathscr{R}^{*}, \quad-\sum_{\boldsymbol{\xi} \in \mathscr{R} *}\left[(i \boldsymbol{k}+i \boldsymbol{\zeta}) \cdot \boldsymbol{C}_{\boldsymbol{\zeta}-\boldsymbol{\xi}} \cdot(i \boldsymbol{k}+i \boldsymbol{\xi})+\omega^{2} \rho_{\zeta-\boldsymbol{\xi}} \boldsymbol{I}\right] \cdot \tilde{\boldsymbol{u}}_{\boldsymbol{\xi}}=\tilde{\boldsymbol{f}}_{\zeta},
$$

where $\boldsymbol{C}_{\boldsymbol{\xi}}$ and $\rho_{\boldsymbol{\xi}}$, for $\boldsymbol{\xi} \in \mathscr{R}^{*}$, are the Fourier series components of $\boldsymbol{C}(\boldsymbol{x})$ and $\rho(\boldsymbol{x})$.

Keeping that in mind, we know that the effective displacement is calculated for each plane wave $\tilde{\boldsymbol{f}}_{\boldsymbol{\xi}} e^{i(\boldsymbol{k}+\boldsymbol{\xi}) \cdot \boldsymbol{x}}$ independently according to the same representation equation (2.19) averaged with respect to $\boldsymbol{y}$. However, since $\boldsymbol{g}$ is defined with respect to $\boldsymbol{k}$ and is now applied to a body force of wavenumber $\boldsymbol{k}+\boldsymbol{\xi}$, two phase factors appear so as to yield

$$
\forall \boldsymbol{\xi} \in \mathscr{R}^{*}, \quad \tilde{\boldsymbol{U}}_{\boldsymbol{\xi}}=\left\langle\left\langle e^{i \boldsymbol{\xi} \cdot \boldsymbol{y}} \boldsymbol{g}(\boldsymbol{y}, \boldsymbol{x}) e^{i \boldsymbol{\xi} \cdot \boldsymbol{x}}\right\rangle\right\rangle \cdot \tilde{\boldsymbol{f}}_{\boldsymbol{\xi}}
$$

A simple matrix inversion yields then the effective motion equation:

$$
\forall \boldsymbol{\xi} \in \mathscr{R}^{*}, \quad\left\langle\left\langle e^{i \boldsymbol{\xi} \cdot \boldsymbol{y}} \boldsymbol{g}(\boldsymbol{y}, \boldsymbol{x}) e^{i \boldsymbol{\xi} \cdot \boldsymbol{x}}\right\rangle\right\rangle^{-1} \cdot \tilde{\boldsymbol{U}}_{\xi}=\tilde{\boldsymbol{f}}_{\boldsymbol{\xi}}
$$

which is the Fourier-series counterpart of equation (2.4).

Comparing equations (2.30) and (2.31) at the microscopic level with equations (2.32) and (2.33) at the effective one, it becomes clear what Willis effective behavior is about. It keeps the same set of kinematical degrees of freedom, unreduced, called $\left\{\tilde{\boldsymbol{u}}_{\xi}\right\}_{\boldsymbol{\xi} \in \mathscr{R}^{*}}$ in the microscopic description and $\left\{\tilde{\boldsymbol{U}}_{\boldsymbol{\xi}}\right\}_{\boldsymbol{\xi} \in \mathscr{R}^{*}}$ in the effective one, and substitutes/approximates the coupled system (2.31) with the decoupled one (2.31) by extracting the diagonal of the microscopic representation equation (2.30) in order to yield the effective one (2.32). Such extraction procedure has the benefit of yielding a homogeneous substitution medium. However, decoupling degrees of freedom is not always legitimate at least for two reasons.

On one hand, it leads to violating our generalized version of Hill-Mandel lemma unless the first homogenizability condition is respected. Recall that this first condition does reduce the kinematical degrees of freedom and only allows for a restricted set of wavenumbers (see condition (2.8) and in particular (2.10)).

On the other hand, given a free wave of the form (2.29), the components $\tilde{\boldsymbol{u}}_{\boldsymbol{\xi}}$ shape one another through (2.31) with $\tilde{\boldsymbol{f}}_{\boldsymbol{\zeta}}=\mathbf{0}$. However, the effective components $\tilde{\boldsymbol{U}}_{\boldsymbol{\xi}}$ are completely independent as can be seen from (2.33). The effective components can be weighted (since body forces are null) and combined by linearity in order to yield an infinite number of effective free-wave solutions only one of which can be said to be physical while all the others are artifacts of the homogenization method. 
The physical effective free wave can be selected as discussed when introducing the third homogenizability condition in $\S 2.4 .3$.

It becomes clear then that there is no escaping some homogenizability condition that should reduce, or guide the selection of, the effective kinematical degrees of freedom for the extraction procedure to avoid the mentioned inconsistencies.

\section{An application: 1D discrete systems}

Hereafter, we introduce a 1D discrete periodic model which can be viewed as a variant of Born's model for sodium chloride considered by Brillouin in his famous work (Brillouin, 1953). This model is studied here as an example to illustrate and to gain insight into the general elastodynamic homogenization theory presented in the foregoing section for periodic media.

\subsection{Problem set-up}

From now on, $\Omega$ is a discrete medium embedded in a $1 \mathrm{D}$ space, and correspondingly $\mathscr{R}$ reduces to a $1 \mathrm{D}$ lattice defined by $\{a \boldsymbol{b}, a \in \mathbb{Z}\}$ with $\boldsymbol{b}$ being the only basis vector. For simplicity, $\Omega$ is taken to be a periodic lattice of which a unit cell $T$ is formed of two different masses and two springs of distinct stiffnesses but of the same length as shown in figure 4.

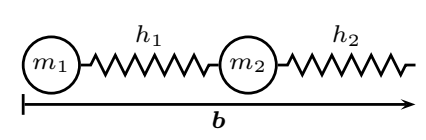

Figure 4: The model unit cell $T$.

In the situation under consideration, the space variable $x$ is discrete. Given the characteristic length $\ell=|T| / 2=\|\boldsymbol{b}\| / 2$ of the unit cell $T$, all positions can be specified by $x=n \ell$ with $n \in \mathbb{Z}$. With no loss of generality, we take $\ell$ to be the unit length, and refer to position $x$ with the integer $n$. Masses $m$ and stiffnesses $h$ are functions of $n \in \mathbb{Z}$. The periodicity of the system dictates that for every position $n$ in $\mathbb{Z}, m_{n+2}=m_{n}$ and $h_{n+2}=h_{n}$. The displacement $u$, velocity $v$ and momentum $p$ are defined pointwisely only for the masses while the strain $\varepsilon$ and stress $\sigma$ make sense only for the springs. Concerning these quantities, we have

$$
\begin{aligned}
\varepsilon_{n}(t) & =u_{n+1}(t)-u_{n}(t), \\
p_{n}(t) & =m_{n} v_{n}(t), \\
\sigma_{n}(t) & =h_{n} \varepsilon_{n}(t) .
\end{aligned}
$$


The motion equation reduces to

$$
\sigma_{n}(t)-\sigma_{n-1}(t)+f_{n}(t)=\dot{p}_{n}(t)
$$

where $f_{n}(t)$ is a concentrated force applied to mass number $n$.

\subsection{Discrete operators and notations}

As explained in the previous section, a generic field $\phi_{n}(t)$ appropriately defined over $\Omega$ can be expanded into harmonic Bloch waves $\tilde{\phi}_{n} e^{i(k n+\omega t)}$ where $\tilde{\phi}_{n}=\tilde{\phi}_{n+2}$ is said to be 2-periodic. From now on, we can restrict our study to a given mode $(k, \omega)$ and to a unit cell containing two consecutive masses, say mass 1 and mass 2 (as depicted in figure 4). Thus, $\tilde{\phi}_{n}$ can be identified with a $2 \mathrm{D}$ vector:

$$
\tilde{\phi}_{n} \mapsto\left[\begin{array}{c}
\tilde{\phi}_{1} \\
\tilde{\phi}_{2}
\end{array}\right]
$$

The motion equation can be written in the simple matrix form

$$
|T|[K][\tilde{u}] \equiv\left[\begin{array}{cc}
-\omega^{2} m_{1}+h_{1}+h_{2} & -h_{1} e^{i k}-h_{2} e^{-i k} \\
-h_{2} e^{i k}-h_{1} e^{-i k} & -\omega^{2} m_{2}+h_{1}+h_{2}
\end{array}\right]\left[\begin{array}{c}
\tilde{u}_{1} \\
\tilde{u}_{2}
\end{array}\right]=\left[\begin{array}{c}
\tilde{f} \\
\tilde{f}
\end{array}\right]
$$

The inverse of the above rigidity-like matrix $K$, namely $G=K^{-1}$, is the discrete equivalent of the Green operator $g$. Recall that $K$ and $G$ depend on the frequency $\omega$ and wavenumber $k$.

To derive the effective properties, we apply formulae (2.28). For this, some remarks are useful. The medium under consideration is $1 \mathrm{D}$, so that no distinction is needed between simple contraction $(\cdot)$, double contraction $(:)$ and symmetric tensorial product $\otimes^{s}$. In addition, all operators must be replaced by their discrete 1D equivalent version. In particular, the following substitutions are to be made:

$$
\begin{aligned}
U(x) & \mapsto \frac{\tilde{u}_{1}+\tilde{u}_{2}}{2} e^{i k n}, \\
g(x, y) & \mapsto[G], \\
\langle\langle g\rangle\rangle & \mapsto\langle\langle G\rangle\rangle \equiv \frac{G_{11}+G_{12}+G_{21}+G_{22}}{4}, \\
g \otimes^{s}\left(\nabla_{x}-i k\right): C & \mapsto[G]\left[\begin{array}{cc}
-1 & e^{-i k} \\
e^{-i k} & -1
\end{array}\right]\left[\begin{array}{cc}
h_{1} & 0 \\
0 & h_{2}
\end{array}\right], \\
\langle C\rangle & \mapsto \frac{h_{1}+h_{2}}{2} .
\end{aligned}
$$




\subsection{Effective constitutive relation}

Accounting for the above modifications made for the 1D system under investigation, it is straightforward to obtain from formulae (2.28) its Willis effective constitutive relation as follows ${ }^{10}$

$$
\left[\begin{array}{l}
\Sigma \\
P
\end{array}\right]=\left[\begin{array}{ll}
C^{e} & S^{1} \\
S^{2} & \rho^{e}
\end{array}\right]_{k, \omega}\left[\begin{array}{l}
E \\
V
\end{array}\right]
$$

where

$$
\begin{aligned}
& C_{k, \omega}^{e}=\frac{4 \cos ^{2}\left(\frac{k}{2}\right)-\omega^{2}\left\langle\frac{\langle m\rangle}{h}\right\rangle}{4 \cos ^{2}\left(\frac{k}{2}\right)-\omega^{2} \frac{\langle m\rangle}{\langle h\rangle}}\left\langle\frac{1}{h}\right\rangle^{-1}, \\
& \rho_{k, \omega}^{e}=\frac{4 \cos ^{2}\left(\frac{k}{2}\right)-\omega^{2}\left\langle\frac{\langle h\rangle}{m}\right\rangle^{-1}}{4 \cos ^{2}\left(\frac{k}{2}\right)-\omega^{2} \frac{\langle m\rangle}{\langle h\rangle}}\langle m\rangle, \\
& S_{k, \omega}^{1}=\frac{i \omega \Delta m \Delta h \cos \left(\frac{k}{2}\right)}{4 \cos ^{2}\left(\frac{k}{2}\right)-\omega^{2} \frac{\langle m\rangle}{\langle h\rangle}} \frac{e^{i k / 2}}{2\langle h\rangle} \equiv i \omega \hat{S}_{k, \omega}^{1},
\end{aligned}
$$

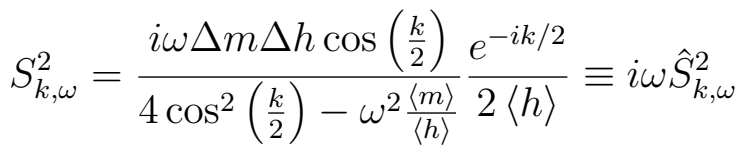

with $\Delta m=m_{1}-m_{2}$ and $\Delta h=h_{1}-h_{2}$.

As $\omega$ goes toward zero, the effective stiffness $C^{e}$ approaches its static limit $\langle 1 / h\rangle^{-1}$ and the effective mass approaches the static effective mass $\langle m\rangle$. At the same time, the coupling terms $S^{1}$ and $S^{2}$ vanish for $\omega=0$ as expected. This also guarantees that no stress is generated from a change of the Galilean frame of reference. It is therefore more correct to say that stresses are coupled with acceleration and not velocity. In the same manner, momenta are coupled with strain rate and not strain. Explicitly, this writes

$$
\left[\begin{array}{l}
\Sigma \\
P
\end{array}\right]=\left[\begin{array}{cc}
C^{e} & 0 \\
0 & \rho^{e}
\end{array}\right]\left[\begin{array}{c}
E \\
V
\end{array}\right]+\left[\begin{array}{cc}
0 & \hat{S}^{1} \\
\hat{S}^{2} & 0
\end{array}\right]\left[\begin{array}{c}
\dot{E} \\
\dot{V}
\end{array}\right]
$$

At last, if the masses $m$ or the stiffnesses $h$ were uniform, the coupling terms would vanish as well. If both masses and stiffnesses are uniform, the initial classical constitutive relation is recovered.

\footnotetext{
${ }^{10}$ We underline the fact that here, unlike the general continuous case, $\Sigma$ is homogeneous to a force, $P$ to a momentum and not to a momentum density, $E$ to a length and $V$ to a velocity. The dimensions of the effective parameters change therefore accordingly.
} 
It is remarkable that the effective medium, of our mass-spring lattice, is also discrete. In fact, the effective motion equation is

$$
\left(e^{i k}-1\right) \Sigma_{k, \omega}+f_{k, \omega}=i \omega P_{k, \omega}
$$

which transforms to the harmonic discrete motion equation in the real (integer) space variable $n$

$$
\Sigma_{n}-\Sigma_{n-1}+f_{n}=i \omega P_{n}
$$

In addition, the effective constitutive parameters are $2 \pi$-periodic functions in the wavenumber $k$. The corresponding convolution kernels are inverse Fourier transforms with respect to $k$ and have therefore a discrete support of step 1 (i.e., the set of integers $\mathbb{Z}$ ). This is similar to the known fact that Fourier transform of a periodic function has a discrete support (hence the use of Fourier series).

Two singularities may appear in the calculations leading to the above expressions of the effective parameters. Indeed, the formulae (2.28) are valid only under two invertibility conditions equivalent to those discussed in (Norris et al., 2012) and reduce, in our discrete case under investigation, to

$$
\operatorname{det} K \neq 0, \quad\langle\langle G\rangle\rangle \neq 0 \text {. }
$$

Let us call an eigenmode, a couple $(k, \omega)$ satisfying the dispersion relation provided by

$$
\operatorname{det} K=0 \text {. }
$$

Eigenmodes allow for non null displacement solutions $u$ even when $f$ is null. Such solutions correspond to free waves. To calculate the effective properties, we may start by avoiding eigenmodes, and then extrapolate by continuity. Hence, we assume that $K$ is invertible ${ }^{11}$ so as to satisfy the first invertibility requirement. This singularity does appear in neither of the denominators of $C^{e}, \rho^{e}, S^{1}$ nor $S^{2}$ which confirms that it is a "removable" singularity as expected.

As for the second condition, the averaged representation equation (2.19) with $\gamma=0$ reduces to

$$
\langle\tilde{u}\rangle=\langle\langle G\rangle\rangle \tilde{f} .
$$

If $\langle\langle G\rangle\rangle=0$ for a couple $(k, \omega)$, then $\langle\tilde{u}\rangle$ and the effective displacement $U$ are null even when the microscopic solution $\tilde{u}$ is non-null. In fact, the effective properties can be said to be divergent for such couples in the sense that the material does not respond to the solicitation $f$ as if it had an infinite inertia. This singularity is not "removable" and is visible in the common denominator of the expressions of $C^{e}, \rho^{e}$, $S^{1}$ and $S^{2}$.

\footnotetext{
${ }^{11}$ This is equivalent to avoiding the singularities of $\boldsymbol{g}$ in the continuous case.
} 
It will be useful to define some dimensionless parameters. As $k$ is measured in $\ell^{-1}$ unit, it can be considered as being dimensionless. Let $\omega_{\circ}$ be the characteristic frequency defined by

$$
\omega_{\circ}^{2}=4\left\langle\frac{\langle m\rangle}{h}\right\rangle^{-1}
$$

and let be introduced the normalized frequency $\nu=\omega / \omega_{0}$. At last, define the following structural dimensionless parameters

$$
\delta_{m}=\frac{1}{\langle m\rangle\langle 1 / m\rangle}, \quad \delta_{h}=\frac{1}{\langle h\rangle\langle 1 / h\rangle} \quad \text { and } \quad \delta=\delta_{h} \delta_{m}
$$

Then, for example, the effective stiffness and effective mass can be simply and compactly written as

$$
C_{k, \omega}^{e}=\frac{\cos ^{2}\left(\frac{k}{2}\right)-\nu^{2}}{\cos ^{2}\left(\frac{k}{2}\right)-\delta_{h} \nu^{2}}\left\langle\frac{1}{h}\right\rangle^{-1} \quad \text { and } \quad \rho_{k, \omega}^{e}=\frac{\cos ^{2}\left(\frac{k}{2}\right)-\delta \nu^{2}}{\cos ^{2}\left(\frac{k}{2}\right)-\delta_{h} \nu^{2}}\langle m\rangle .
$$

\subsection{Effective impedance and dispersion curves}

Determination of the effective displacement response $U$ to a solicitation $f$ does not entail knowing the whole effective properties. Indeed, a direct relation between $U$ and $f$ such as

$$
z_{k, \omega} U=f
$$

can be found, where $z=\langle\langle G\rangle\rangle^{-1}$ is an effective impedance given by

$$
z_{k, \omega}=\left|e^{i k}-1\right|^{2} C^{e}+i \omega\left(e^{-i k}-1\right) S^{1}+i \omega\left(e^{i k}-1\right) S^{2}-\omega^{2} \rho^{e}
$$

which can be further specified as

$$
z_{k, \omega}=\frac{2 m_{1} m_{2} \omega^{4}-2\left(h_{1}+h_{2}\right)\left(m_{1}+m_{2}\right) \omega^{2}+8 h_{1} h_{2} \sin ^{2}(k)}{4\left(h_{1}+h_{2}\right) \cos ^{2}\left(\frac{k}{2}\right)-\left(m_{1}+m_{2}\right) \omega^{2}} .
$$

In terms of the dimensionless parameters $k$ and $\nu$, we have

$$
z_{k, \omega}=\left\langle\frac{1}{h}\right\rangle^{-1} \frac{4 \delta \nu^{4}-4 \nu^{2}+\sin ^{2}(k)}{\cos ^{2}\left(\frac{k}{2}\right)-\delta_{h} \nu^{2}} .
$$

The impedance $z$ is always real for similar 1D lattices with $|T|>2$ and, in this particular case $|T|=2$, the contributions of $S^{1}$ and of $S^{2}$ annihilate each other and disappear in the above expression. The coupling terms still affect effective stress and momentum. However, one must consider more complex situations $(|T|>2)$ or more general loadings $(\gamma \neq 0)$ to study their effect on the motion equation. 
Effective eigenmodes are points of the curve $\left\{z_{k, \omega}=0\right\}$ while microscopic eigenmodes are points of the curve $\left\{\operatorname{det} K_{k, \omega}=0\right\}$. We have proved that microscopic solutions are transformed into effective solutions under the same loading. Therefore, a point $(k, \omega)$ of $\left\{\operatorname{det} K_{k, \omega}=0\right\}$ indicates the existence of a microscopic non-null free wave solution $u$ which is transformed into an effective free wave solution $U$ meaning that $(k, \omega)$ is a point of $\left\{z_{k, \omega}=0\right\}$ except when $U=0$. This is: except when $(k, \omega)$ satisfies also $\left\langle\left\langle G_{k, \omega}\right\rangle\right\rangle=0$. To sum up, we have

$$
\left\{z_{k, \omega}=0\right\}=\left\{\operatorname{det} K_{k, \omega}=0\right\} \backslash\left\{\left\langle\left\langle G_{k, \omega}\right\rangle\right\rangle=0\right\} .
$$

Given the effective dispersion curve, one can recover the microscopic dispersion curve by continuous extrapolation unless $\left\{\operatorname{det} K_{k, \omega}=0\right\}$ and $\left\{\left\langle\left\langle G_{k, \omega}\right\rangle\right\rangle=0\right\}$ coincide $^{12}$ over a non-empty open subset of $T^{*}$.

The dispersion relation is characterized by

$$
\left\langle\frac{\langle h\rangle}{m}\right\rangle^{-1} \omega^{4}-4 \omega^{2}+4\left\langle\frac{\langle m\rangle}{h}\right\rangle^{-1} \sin ^{2}(k)=0
$$

which can be written

$$
4 \delta \nu^{4}-4 \nu^{2}+\sin ^{2}(k)=0 .
$$

The solutions of this equation parametrized by $\delta$ are plotted in figure 5 . The parameter $\delta$ can be interpreted as a measure of the heterogeneity contrast in the medium. In fact, the arithmetic and harmonic inequalities imply that

$$
1 /\langle 1 / h\rangle \leq\langle h\rangle, \quad 1 /\langle 1 / m\rangle \leq\langle m\rangle
$$

with the equality attained in the first inequality (or the second inequality) if and only if stiffnesses (or masses) are uniform. Therefore, $\delta$ is comprised between 0 and 1 with $\delta=0$ for an extremely high contrast medium (one mass is infinite for example) and $\delta=1$ only for a homogeneous medium.

\footnotetext{
${ }^{12}$ In our case, this intersection has in it a single point at most.
} 


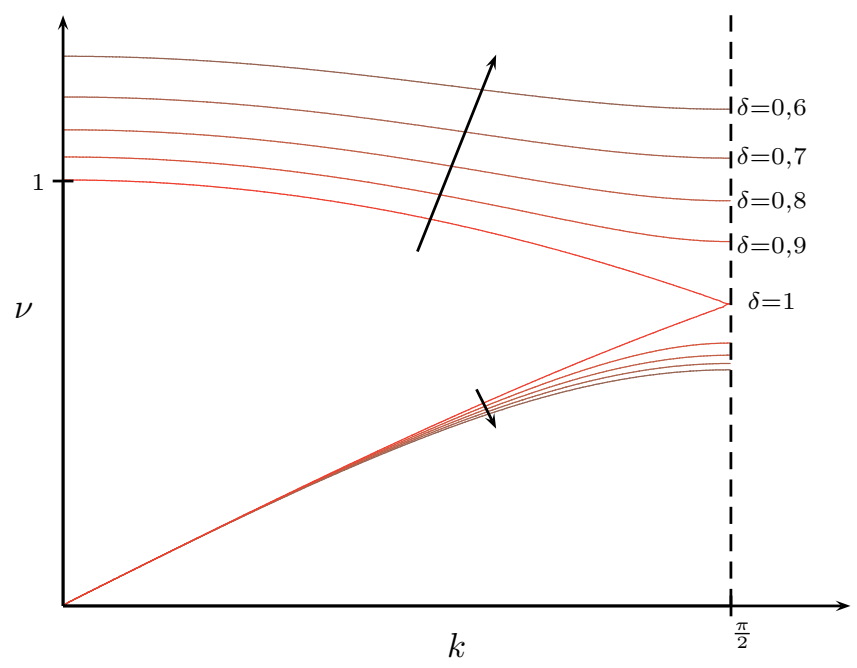

Figure 5: Dispersion curve family (equation (3.2)) indexed by $\delta$ (decreasing with the arrows). Since the homogeneous medium $(\delta=1)$ is treated as a 2-periodic medium, its dispersion curve is folded into the first Brillouin zone of a 2-periodic medium and has only one branch. All other curves have two branches: an acoustic one of low frequency $\nu$, and an optical one of higher frequency $\nu$.

Over the frequency domain or $\nu$-space, there are two prohibited zones which prevent free waves from propagating (see figure 6):

- beyond a cut-off frequency $\nu_{c}$ defined by

$$
\nu_{c}^{2}=\frac{1}{\delta}
$$

which corresponds to

$$
\omega_{c}^{2}=\frac{\omega_{\circ}^{2}}{\delta}=4\left\langle\frac{\langle h\rangle}{m}\right\rangle .
$$

For a homogeneous medium, we recover $\nu_{c}=1$ which is the classical cut-off frequency

$$
\omega=\omega_{\circ}=2 \sqrt{\frac{h}{m}}
$$

- Over a bounded range (a "bandgap", see (Quéré, 1988) in the context of solid state physics) between the frequencies $\nu_{\text {low }}$ and $\nu_{\text {high }}$ given by

$$
\begin{gathered}
\nu_{\text {low }}^{2}=\frac{1-\sqrt{1-\delta}}{2 \delta}, \\
\nu_{\text {high }}^{2}=\frac{1+\sqrt{1-\delta}}{2 \delta} .
\end{gathered}
$$


The higher the contrast is, the wider the bandgap is. For a homogeneous medium, the bandgap disappears.

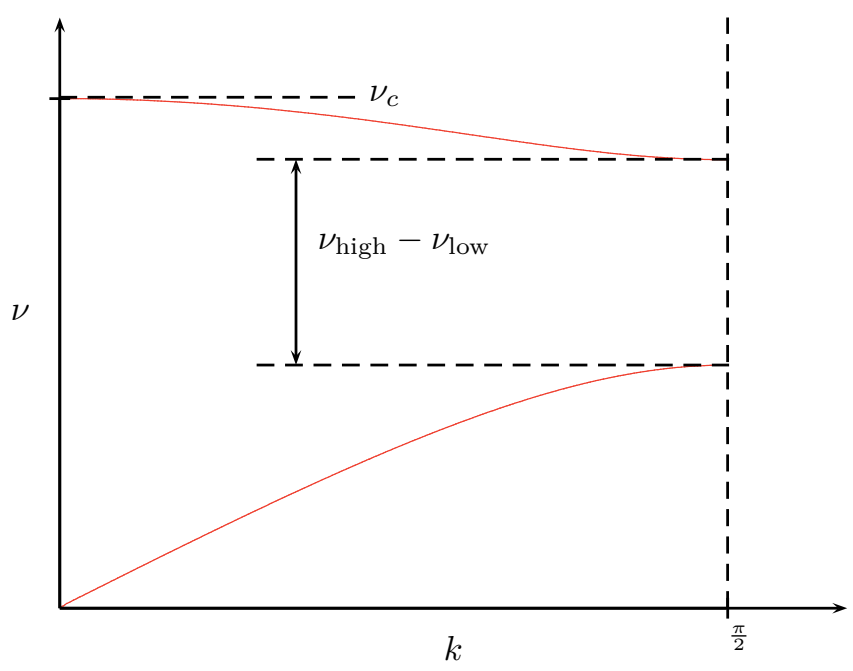

Figure 6: Bandgap and cut-off frequency $(\delta=0.7)$.

It is known and easy to see that, at low frequencies, the effective mass is the arithmetic mean of masses. Let us then investigate, through direct analysis, the high frequency behavior of the effective mass through two examples $\nu=\nu_{c}$ and $\nu \rightarrow \infty$.

- The exact solution for the eigenmode $\left(k=0, \nu=\nu_{c}\right)$ is $u(x, t)=\tilde{u}(x) e^{i \omega t}$. Since $\tilde{u}$ is 2 -periodic, the eigenmode $\left(0, \nu_{c}\right)$ decouples the medium into two rigid subsystems (even indexed masses and odd indexed masses) connected by two springs and oscillating in two opposite directions (so as not to violate Newton's first law, see figure 7). It is easy then to see that $E=0$ since $k=0$, that $P=0$ since $u_{1} / u_{2}=-m_{2} / m_{1}$ and that $V \neq 0$ for $m_{1} \neq m_{2}$. Therefore, $\rho_{k=0, \nu=\nu_{c}}^{e}=0$.

- For even higher frequencies $\nu \rightarrow \infty$, no free waves can propagate meaning that, given a force field $\tilde{f}$, masses will oscillate as if independent of each other. We then have $-\omega^{2} m_{1} \tilde{u}_{1}=-\omega^{2} m_{2} \tilde{u}_{2}=\tilde{f}$ meaning that $i \omega\langle\tilde{u}\rangle=\langle 1 / m\rangle \tilde{f} / i \omega$ and $\rho_{k, \nu \rightarrow \infty}^{e}=1 /\langle 1 / m\rangle$.

These two examples, along with the quasi-static case, show that the time nonlocality which is caused by the $\omega$ or $\nu$-dependency of the effective mass, among others, can 
be interpreted as the effect, over effective mass, of changing modes of oscillation (in/out of phase for example) of internal degrees of freedom.

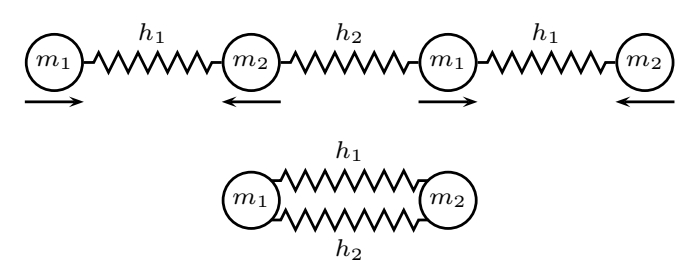

Figure 7: The mode $\left(k=0, \nu=\nu_{c}\right)$ (top) and the two-body problem that it reduces to (bottom). The arrows illustrate the direction of the velocity at a given time.

Finally, remark that the dispersion curve for $\delta_{h}=1$ where stiffnesses are uniform is nothing else than the dispersion curve calculated by Brillouin for Born's model in (Brillouin, 1953).

\subsection{Homogenizability conditions illustrated}

For the 1D discrete medium under consideration, the second homogenizability condition (2.10) is simply

$$
-\frac{\pi}{2}<k<\frac{\pi}{2}
$$

As the gradient operator is replaced by its discrete version, the third homogenizability condition becomes

$$
\omega^{2} \lesssim 4 \max \left(\frac{h}{m}\right) \sin ^{2}\left(\frac{k}{2}\right)
$$

or, in terms of the reduced frequency $\nu$,

$$
\nu^{2} \lesssim\left\langle\frac{\langle m\rangle}{h}\right\rangle \max \left(\frac{h}{m}\right) \sin ^{2}\left(\frac{k}{2}\right) .
$$

Now, we can easily check that

$$
\left\langle\frac{\langle m\rangle}{h}\right\rangle \max \left(\frac{h}{m}\right) \leq\left\langle\frac{\langle m\rangle}{h}\right\rangle \frac{\max (h)}{\min (m)} \leq\left\langle\frac{\langle m\rangle}{h}\right\rangle\langle h\rangle\left\langle\frac{1}{m}\right\rangle=\delta^{-1}
$$

which leads to

$$
\delta \nu^{2} \lesssim \sin ^{2}\left(\frac{k}{2}\right)
$$


Finally, solving the dispersion equation (3.2) for $\sin ^{2}(k / 2)$, we obtain

$$
\delta\left(1+\sqrt{1+4 \nu^{2}\left(\delta \nu^{2}-1\right)}\right) \lesssim 2\left(1-\delta \nu^{2}\right)
$$

under both eigenmodes existence conditions previously discussed, i.e.,

$$
\nu^{2} \leq 1 / \delta \quad \text { and } \quad 4 \nu^{2}\left(1-\delta \nu^{2}\right) \leq 1
$$

This means that $\nu$ must be below the cut-off frequency and outside the bandgap. The above inequalities are illustrated in figure 8. The homogenizable frequency domain covers the whole first branch of the dispersion curve and the low-frequency part of the second branch. As this simple 1D medium has no internal resonances, the first optical branch is not homogenizable which agrees with the observation made by Srivastava and Nemat-Nasser (2014). Borrowing Brillouin's interpretation, the combined homogenizability conditions state that consecutive masses should not oscillate in an obvious out-of-phase fashion. 


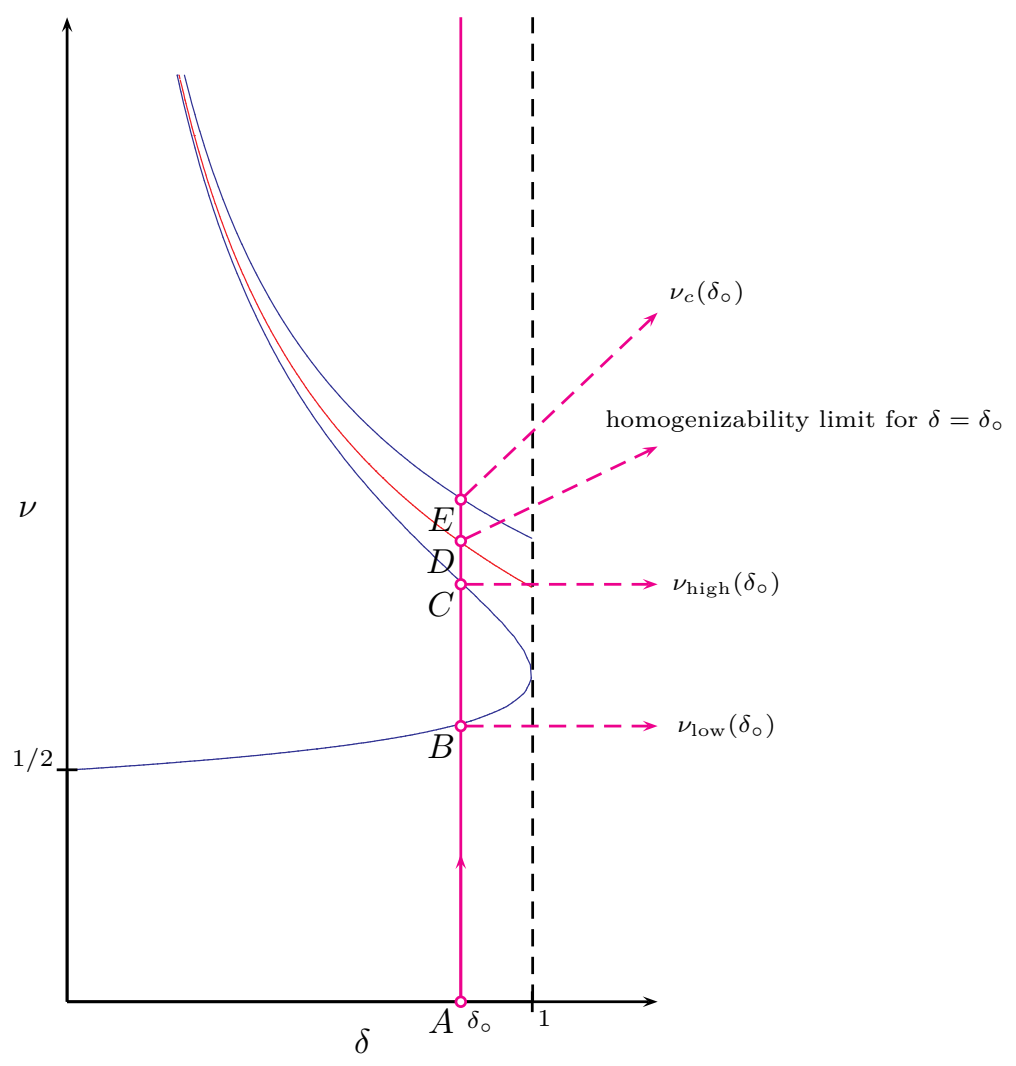

Figure 8: The homogenizability condition (3.3) in the $(\delta, \nu)$-space. Starting from point $A$ at $\left(\nu=0, \delta=\delta_{\circ}\right)$ and following the increasing $\nu$, we scan the acoustic branch of the dispersion curve, then we meet point $B$ where the bandgap begins. At point $C$ the bandgap ends and the optical branch starts. The point $D$ indicates the end of the homogenizable frequency domain. Finally, the cut-off frequency is attained at point $E$. The inequalities (3.4) are satisfied between $A$ and $B$ and between $C$ and $E$ while the homogenizability condition (3.3) is satisfied between $A$ and $D$.

Figure 9 shows an example of an exact high frequency free wave solution where a macroscopic description appears to be out of context. Therein, the effective wavelength satisfies the second homogenizability condition. The large difference between the amplitudes of the effective and microscopic waves indicates that the dominant component is the short-wavelength one. This is due to the chosen high frequency that violates the third homogenizability condition. In figure 10 is given an example of an exact low frequency free wave solution where the macroscopic behavior is rather obvious. The dominant component is clearly the effective long-wavelength one due to the low frequency setting in agreement with the third homogenizability condition. For these examples, details (masses, stiffnesses, time, ...) which are not important from the physical standpoint are not specified. 


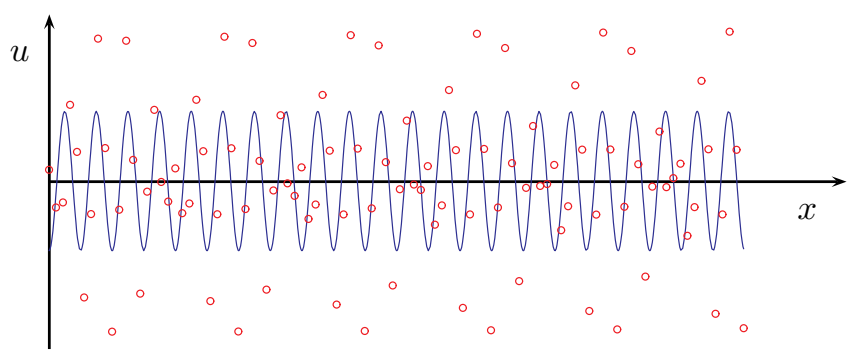

Figure 9: In small circles, an exact high frequency free wave solution: the (real) displacement of 100 consecutive masses. The continuous line draws the corresponding effective plane wave.

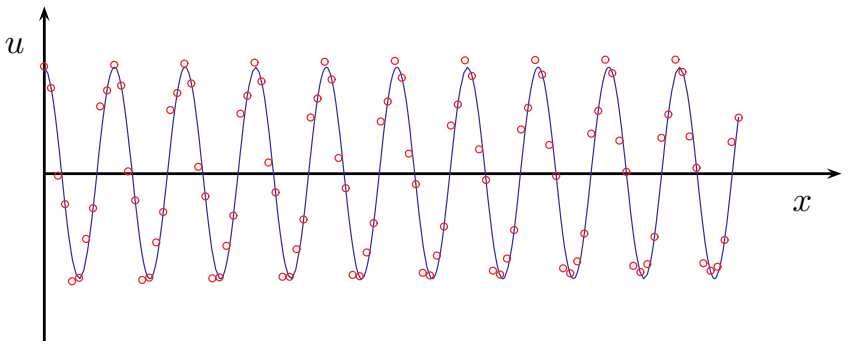

Figure 10: In small circles, an exact low frequency free wave solution: the (real) displacement of 100 consecutive masses. The continuous line draws the corresponding effective plane wave.

\section{Concluding remarks}

The elastodynamic homogenization theory of composites, initiated by J.R. Willis more than thirty years ago, escaping due attention during a long period, and developed recently by him and other researchers, has been carefully and systematically revisited in the present work for periodic media. The theory of Willis is general and exact, leading to an effective elastodynamic constitutive law which is nonlocal in time and space and takes a form quite different from and much more complicated than the usual constitutive laws in classical elastodynamics. Many important questions concerning this theory and its applications remain 
open. In the present paper, three ones have been addressed and studied. First, in spite of its mathematical generality and exactness, the corresponding physical validity (or suitableness) domain remains far from being clarified. Aiming mainly at giving an answer to this important question, the present work has established three necessary conditions for the application of that theory to be physically sound. Second, even though the theory of Willis has recently been numerically studied and applied to some relatively simple periodic cases (see, e.g., papers by NematNasser and Srivastava (2011) and Srivastava and Nemat-Nasser (2011)), other general, more robust, numerical methods are still lacking for its implementation and applications in more involved periodic situations. This observation has led us to express the frequency- and wavenumber-dependent parameters characterizing the effective constitutive law in the spirit of micromechanics and in an explicit way, so that a versatile numerical method, such as the finite element one, can be directly constructed and applied to compute them. Third, the physical complicatedness and implications of the theory of Willis appear to be far from being fully understood. For this reason, in the present work, a very simple periodic discrete system has been analytically, exactly and exhaustively investigated to get insights into that theory.

Among other open questions concerning the elastodynamic homogenization theory of Willis for composites, the following ones appear to be particularly significant:

1. In the literature, several approaches based on asymptotic analysis have been proposed to study elastodynamic homogenization (Andrianov et al., 2008; Auriault and Bonnet, 1985; Auriault and Boutin, 2012; Boutin, 1996; Boutin and Auriault, 1993; Craster et al., 2010). What are the relations of the theory of Willis to these approaches? This fundamental problem has recently been investigated by us and the results obtained will be presented in a forthcoming paper.

2. The elastodynamic homogenization theory proposed by Willis holds formally both for linear elasticity and linear viscoelasticity (Willis, 2009, 2011). Up to now, all the detailed studies and numerical examples reported in the literature to develop and illustrate this theory have however been limited to linear elasticity. How to concretely apply the theory to the more complex case of linear viscoelasticity remains a basic question to be answered.

3. The theory of Willis is formally valid for both periodically and randomly inhomogeneous materials (Willis, 2009, 2011). Nevertheless, due to the fact that randomly inhomogeneous materials are much more complicated than periodically inhomogeneous ones, all the relevant detailed studies carried out in the literature have restricted themselves to the periodic situation. The more involved case of randomly inhomogeneous materials remains largely to 
be studied and explored. Such a study should be done in a near future owing to the theoretical and practical importance of random composites.

4. The extension of Willis' theory to nonlinear composites, even to very simple ones, seems to have never been done. This extension appears to be very challenging and difficult both from the conceptual and technical standpoints.

\section{References}

Amirkhizi, A.V., Nemat-Nasser, S., 2008. Microstructurally-based homogenization of electromagnetic properties of periodic media. Comptes Rendus Mécanique $336,24-33$.

Andrianov, I.V., Bolshakov, V.I., Danishevs'kyy, V.V., Weichert, D., 2008. Higher order asymptotic homogenization and wave propagation in periodic composite materials. Proceedings of the Royal Society A: Mathematical, Physical and Engineering Sciences 464, 1181-1201.

Auriault, J.L., Bonnet, G., 1985. Dynamics of periodic elastic composites (Dynamique des composites elastiques periodiques). Archiwum Mechaniki Stosowanej $37,269-284$.

Auriault, J.L., Boutin, C., 2012. Long wavelength inner-resonance cut-off frequencies in elastic composite materials. International Journal of Solids and Structures 49, 3269-3281.

Bonnet, M., 1995. Équations intégrales et éléments de frontière: applications en mécanique des solides et des fluides. Collection Sciences et techniques de l'ingénieur. Eyrolles ed., CNRS éd.

Boutin, C., 1996. Microstructural effects in elastic composites. International Journal of Solids and Structures 33, 1023-1051.

Boutin, C., Auriault, J.L., 1993. Rayleigh scattering in elastic composite materials. International Journal of Engineering Science 31, 1669-1689.

Brillouin, L., 1953. Wave propagation in periodic structures: Electric filters and crystal lattices. Dover phoenix editions, Dover Publications.

Chen, H., Chan, C.T., 2010. Acoustic cloaking and transformation acoustics. Journal of Physics D: Applied Physics 43, 113001.

Craster, R.V., Kaplunov, J., Pichugin, A.V., 2010. High-frequency homogenization for periodic media. Proceedings of the Royal Society A: Mathematical, Physical and Engineering Sciences 466, 2341-2362. 
Fietz, C., Shvets, G., 2010. Current-driven metamaterial homogenization. Physica B: Condensed Matter 405, 2930-2934.

Gazalet, J., Dupont, S., Kastelik, J.C., Rolland, Q., Djafari-Rouhani, B., 2013. A tutorial survey on waves propagating in periodic media: Electronic, photonic and phononic crystals. Perception of the Bloch theorem in both real and Fourier domains. Wave Motion 50, 619-654.

Lee, J.H., Singer, J.P., Thomas, E.L., 2012. Micro-/nanostructured mechanical metamaterials. Advanced materials 24, 4782-4810.

Liu, A.P., Zhu, R., Liu, X.N., Hu, G.K., Huang, G.L., 2012. Multi-displacement microstructure continuum modeling of anisotropic elastic metamaterials. Wave Motion 49, 411-426.

Liu, Z., Zhang, X., Mao, Y., Zhu, Y.Y., Yang, Z., Chan, C.T., Sheng, P., 2000. Locally resonant sonic materials. Science (New York, N.Y.) 289, 1734-6.

Milton, G.W., Briane, M., Willis, J.R., 2006. On cloaking for elasticity and physical equations with a transformation invariant form. New Journal of Physics 248, 248-248.

Milton, G.W., Willis, J.R., 2007. On modifications of Newton's second law and linear continuum elastodynamics. Proceedings of the Royal Society A: Mathematical, Physical and Engineering Sciences 463, 855-880.

Milton, G.W., Willis, J.R., 2010. Minimum variational principles for time-harmonic waves in a dissipative medium and associated variational principles of HashinShtrikman type. Proceedings of the Royal Society A: Mathematical, Physical and Engineering Sciences 466, 3013-3032.

Nemat-Nasser, S., Srivastava, A., 2011. Overall dynamic constitutive relations of layered elastic composites. Journal of the Mechanics and Physics of Solids 59, 1953-1965.

Nemat-Nasser, S., Srivastava, A., 2013. Bounds on effective dynamic properties of elastic composites. Journal of the Mechanics and Physics of Solids 61, 254-264.

Nemat-Nasser, S., Willis, J.R., Srivastava, A., Amirkhizi, A.V., 2011. Homogenization of periodic elastic composites and locally resonant sonic materials. Physical Review B 83, 1-8.

Norris, A.N., 2008. Acoustic cloaking theory. Proceedings of the Royal Society A: Mathematical, Physical and Engineering Sciences 464, 2411-2434. 
Norris, A.N., Shuvalov, A.L., 2011. Elastic cloaking theory. Wave Motion 48, $525-538$.

Norris, A.N., Shuvalov, A.L., Kutsenko, A.A., 2012. Analytical formulation of threedimensional dynamic homogenization for periodic elastic systems. Proceedings of the Royal Society A: Mathematical, Physical and Engineering Sciences 468, $1629-1651$.

Pham, K., Kouznetsova, V., Geers, M., 2013. Transient computational homogenization for heterogeneous materials under dynamic excitation. Journal of the Mechanics and Physics of Solids 61, 2125-2146.

Quéré, Y., 1988. Physique des matériaux: cours et problèmes. X Ecole Polytechnique, Edition Marketing.

Shuvalov, A.L., Kutsenko, A.A., Norris, A.N., Poncelet, O., 2011. Effective Willis constitutive equations for periodically stratified anisotropic elastic media. Proceedings of the Royal Society A: Mathematical, Physical and Engineering Sciences 467, 1749-1769.

Simovski, C.R., 2007. Bloch material parameters of magneto-dielectric metamaterials and the concept of Bloch lattices. Metamaterials 1, 62-80.

Smyshlyaev, V.P., Cherednichenko, K.D., 2000. On rigorous derivation of strain gradient effects in the overall behaviour of periodic heterogeneous media. Journal of the Mechanics and Physics of Solids 48, 1325-1357.

Srivastava, A., Nemat-Nasser, S., 2011. Overall dynamic properties of threedimensional periodic elastic composites. Proceedings of the Royal Society A: Mathematical, Physical and Engineering Sciences 468, 269-287.

Srivastava, A., Nemat-Nasser, S., 2014. On the limit and applicability of dynamic homogenization. Wave Motion doi:10.1016/j.wavemoti.2014.04.003.

Willis, J.R., 1980a. A polarization approach to the scattering of elastic waves-I. Scattering by a single inclusion. Journal of the Mechanics and Physics of Solids $28,287-305$.

Willis, J.R., 1980b. A polarization approach to the scattering of elastic waves-II. Multiple scattering. Journal of the Mechanics and Physics of Solids 28, 307-327.

Willis, J.R., 1981. Variational principles for dynamic problems for inhomogeneous elastic media. Wave Motion 3, 1-11. 
Willis, J.R., 1985. The nonlocal influence of density variations in a composite. International Journal of Solids and Structures 21, 805-817.

Willis, J.R., 1997. Dynamics of composites, in: Suquet, P. (Ed.), Continuum Micromechanics. Springer-Verlag New York, Inc., pp. 265-290.

Willis, J.R., 2009. Exact effective relations for dynamics of a laminated body. Mechanics of Materials 41, 385-393.

Willis, J.R., 2011. Effective constitutive relations for waves in composites and metamaterials. Proceedings of the Royal Society A: Mathematical, Physical and Engineering Sciences 467, 1865-1879.

Willis, J.R., 2012. The construction of effective relations for waves in a composite. Comptes Rendus Mécanique 340, 181-192.

\section{Appendix A. Bloch theorem by a scattering analysis}

The Bloch form (2.1) is not surprising and arises naturally in the following analysis. Let $\boldsymbol{f}$ be some plane wave loading of $\Omega$. The field $\boldsymbol{f}$ can be thought of as a body force, some incident wave or any other loading of the medium. Let $\boldsymbol{k}_{I} \in \mathscr{E}^{*}$ be its wavenumber. By $\mathscr{R}$-periodicity of the microstructure, the perturbation $\boldsymbol{f}$ will interact with the scatterer (an inclusion or an interface for example) placed at point $\boldsymbol{x}_{\circ} \in T$ in the same manner it does with all scatterers placed at $\boldsymbol{x}_{\circ}+\boldsymbol{r}$ for all $\boldsymbol{r} \in \mathscr{R}$. The same amplitude $\boldsymbol{u}_{\boldsymbol{k}_{S}}\left(\boldsymbol{x}_{\circ}\right)$ is then scattered in the direction $\boldsymbol{k}_{S} \in \mathscr{E} *$ from all points $\left\{\boldsymbol{x}_{\circ}+\boldsymbol{r}, \boldsymbol{r} \in \mathscr{R}\right\}$ with a corresponding phase shift of

$$
\boldsymbol{k}_{S} \cdot\left(\boldsymbol{x}_{\circ}+\boldsymbol{r}-\boldsymbol{x}_{\circ}\right)-\boldsymbol{k}_{I} \cdot\left(\boldsymbol{x}_{\circ}+\boldsymbol{r}-\boldsymbol{x}_{\circ}\right)=\left(\boldsymbol{k}_{S}-\boldsymbol{k}_{I}\right) \cdot \boldsymbol{r} .
$$

The scattered field, from the set of scatterers $\left\{\boldsymbol{x}_{\circ}+\boldsymbol{r}, \boldsymbol{r} \in \mathscr{R}\right\}$, in the direction $\boldsymbol{k}_{S}$ is then the sum over $\boldsymbol{r} \in \mathscr{R}$ of $\boldsymbol{u}_{\boldsymbol{k}_{S}}\left(\boldsymbol{x}_{\circ}\right) e^{\left(\boldsymbol{k}_{S}-\boldsymbol{k}_{I}\right) \cdot \boldsymbol{r}}$ which interferes destructively unless $\boldsymbol{k}_{S}-\boldsymbol{k}_{I}$ is a reciprocal lattice wavenumber: $\boldsymbol{k}_{S}-\boldsymbol{k}_{I}=\boldsymbol{\xi} \in \mathscr{R}^{*}$. Therefore, at a given point $\boldsymbol{x}, \boldsymbol{u}(\boldsymbol{x})$ is a sum of contributions from all scatterers $\boldsymbol{x}_{\circ} \in T$ coming from directions $\boldsymbol{k}_{S}=\boldsymbol{k}_{I}+\boldsymbol{\xi}$, for $\boldsymbol{\xi} \in \mathscr{R}^{*}$ (since the contribution of other directions sums to zero):

$$
\begin{aligned}
& \boldsymbol{u}(\boldsymbol{x})=\sum_{\boldsymbol{\xi} \in \mathscr{R}^{*}} \int_{T} \boldsymbol{u}_{\boldsymbol{k}_{S}}\left(\boldsymbol{x}_{\circ}\right) e^{\boldsymbol{k}_{S} \cdot\left(\boldsymbol{x}-\boldsymbol{x}_{\circ}\right)} \mathrm{d} \boldsymbol{x}_{\circ} \\
&=\sum_{\boldsymbol{\xi} \in \mathscr{R}^{*}} \int_{T} \boldsymbol{u}_{\boldsymbol{k}_{S}}\left(\boldsymbol{x}_{\circ}\right) e^{\left(\boldsymbol{k}_{I}+\boldsymbol{\xi}\right) \cdot\left(\boldsymbol{x}-\boldsymbol{x}_{\circ}\right)} \mathrm{d} \boldsymbol{x}_{\circ} \\
&=\left[\sum_{\boldsymbol{\xi} \in \mathscr{R}^{*}}\left(\int_{T} \boldsymbol{u}_{\boldsymbol{k}_{S}}\left(\boldsymbol{x}_{\circ}\right) e^{-\left(\boldsymbol{k}_{I}+\boldsymbol{\xi}\right) \cdot \boldsymbol{x}_{\circ}} \mathrm{d} \boldsymbol{x}_{\circ}\right) e^{\boldsymbol{\xi} \cdot \boldsymbol{x}}\right] e^{\boldsymbol{k}_{I} \cdot \boldsymbol{x}},
\end{aligned}
$$


which is nothing else than the Fourier series expansion of some $\mathscr{R}$-periodic field multiplied by an exponential factor of $e^{\boldsymbol{k}_{I} \cdot \boldsymbol{x}}$ for some "incident" wavenumber $\boldsymbol{k}_{I} \in \mathscr{E}^{*}$, i.e., a Bloch wave. 